\title{
Coronal hole boundaries at small scales
}

\section{SOT view. Magnetic field properties of small-scale transient brightenings in coronal holes ${ }^{\star}$}

\author{
Z. Huang ${ }^{1}$, M. S. Madjarska ${ }^{1,2}$, J. G. Doyle ${ }^{1}$, and D. A. Lamb ${ }^{3}$ \\ 1 Armagh Observatory, College Hill, Armagh BT61 9DG, N. Ireland, UK \\ e-mail: zhu@arm.ac.uk \\ 2 UCL-Mullard Space Science Laboratory, Holmbury St Mary, Dorking, Surrey, RH5 6NT, UK \\ 3 Department of Space Studies, Southwest Research Institute, 1050 Walnut Street Suite 300, Boulder, CO 80302, USA
}

Received 23 July 2012 / Accepted 5 October 2012

\begin{abstract}
Context. We study the magnetic properties of small-scale transients in coronal holes and a few in the quiet Sun identified in X-ray observations and spectroscopic data in two previous papers.

Aims. We aim to investigate the role of small-scale transients in the evolution of the magnetic field in an equatorial coronal hole.

Methods. Two sets of observations of an equatorial coronal hole and another two in quiet-Sun regions were analysed using longitudinal magnetograms taken by the Solar Optical Telescope. An automatic feature tracking program, SWAMIS, was used to identify and track the magnetic features. Each event was then visually analysed in detail.

Results. In both coronal holes and quiet-Sun regions, all brightening events are associated with bipolar regions and are caused by magnetic flux emergence followed by cancellation with the pre-existing and/or newly emerging magnetic flux. In the coronal hole, 19 of 22 events have a single stable polarity which does not change its position in time. In eleven cases this is the dominant polarity. In the coronal hole, the magnetic features with the largest flux are those of the dominant polarity, while the opposite polarity is distributed in weaker features. The number of magnetic features of the dominant polarity is four times grater than the opposite polarity. The supergranulation configuration appears to preserve its general shape during approximately nine hours of observations although the large concentrations (the dominant polarity) in the network did evolve and/or were slightly displaced, and their strength either increased or decreased. The emission fluctuations/radiance oscillations seen in the X-ray bright points are associated with recurring magnetic cancellation in the footpoints. Unique observations of an X-ray jet reveal similar magnetic behaviour in the footpoints, i.e. cancellation of the opposite polarity magnetic flux. We find that the magnetic flux cancellation rate during the jet is much higher than in bright points. Not all magnetic cancellations result in an X-ray enhancement, suggesting that there is a threshold of the amount of magnetic flux involved in a cancellation above which brightening would occur at X-ray temperatures.

Conclusions. Our study demonstrates that the magnetic flux in coronal holes is continuously processed through magnetic reconnection which is responsible for the formation of numerous small-scale transient events. The open magnetic flux forming the coronal-hole phenomenon is largely involved in these transient features. The question of whether this open flux is transported as a result of the formation and evolution of these transient events, however, still remains open.
\end{abstract}

Key words. Sun: corona - Sun: chromosphere - Sun: evolution - magnetic fields - methods: observational

\section{Introduction}

Coronal holes (CHs) are regions on the Sun where the emission of coronal lines is significantly reduced. $\mathrm{CHs}$ are found to be the main source regions of the fast solar wind (Krieger et al. 1973) while coronal hole boundaries are believed to be the regions where the slow solar wind originates (Hundhausen 1977). We first studied the small-scale evolution of CHs and their boundaries using spectroscopic observations from SUMER (Madjarska et al. 2004). This was followed by three studies. The first one investigated dynamic phenomena at the coronal hole boundaries (CHBs) using TRACE (The Transition Region And Coronal Explorer) and EIT (Extreme-ultraviolet

$\star$ Three movies and Appendix A are available in electronic form at http://www. aanda.org
Imaging Telescope) onboard SoHO (Madjarska \& Wiegelmann 2009, hereafter Paper I). The second study by Subramanian et al. (2010, hereafter Paper II) automatically identified X-ray transient brightenings in CHs and the quiet-Sun regions in observations from XRT (The X-Ray Telescope) onboard the Hinode satellite. Next, Madjarska et al. (2012, hereafter Paper III) analysed the plasma properties of all the events which were identified in Paper II having simultaneous spectral observations taken with the EIS (Extreme-ultraviolet Imaging Spectrometer) and SUMER (Solar Ultraviolet Measurements of Emitted Radiation) instruments onboard Hinode and SoHO, respectively. By studying tens of events, the authors found that events in the $\mathrm{CHs}$ and quiet-Sun regions reached similar temperatures and electron densities, but events in $\mathrm{CHs}$ and their boundaries were more dynamic than events in the quiet Sun. Background information on 
coronal holes and an overview on the results of the papers published earlier are given in Madjarska et al. (2012, and the references therein). These studies showed that the brightening events, including coronal bright points (BPs) and jets, are associated with the small-scale dynamics of CHBs.

Coronal BPs are omnipresent in both $\mathrm{CHs}$ and the quiet Sun. They were first identified in soft X-rays (Vaiana et al. 1970) and analysed in detail during the Skylab mission (Golub et al. 1976a,b; Habbal 1992, and references therein). They are small (on average 20"-30") and short-lived (from a few minutes to a few tens of hours) emission enhanced structures observed in the solar corona (Webb et al. 1993, and references therein). They can also be observed in the EUV wavelength bands (Habbal \& Withbroe 1981; Zhang et al. 2001, etc.). BPs represent small-scale loop structures first seen in a unique sequence of high-resolution Naval Research Laboratory (NRL) Skylab spectroheliograms with a spatial resolution of $2^{\prime \prime}$ (Sheeley \& Golub 1979). Sheeley \& Golub (1979) found that BPs consist of two or three miniature loops $(2500 \mathrm{~km}$ in diameter and $12000 \mathrm{~km}$ long) evolving on a time scale of 6 min. Habbal et al. (1990) confirmed this result showing that simultaneously measured peaks of emission in six different lines (emitted from the chromosphere to the corona) were not always co-spatial, implying that the BPs are composed of small-scale loops at different temperatures. The fine structure of BPs consisting of numerous loops was later confirmed by the high-resolution TRACE and now by XRT/Hinode and AIA/SDO observations.

Studies of BPs have shown variations in their radiance lightcurves (Moore et al. 1977; Sheeley \& Golub 1979; Habbal \& Withbroe 1981, etc.). Madjarska et al. (2003) found six minutes radiance variations in SUMER transition region observations of a coronal BP. Ugarte-Urra et al. (2004a) derived intensity oscillations with periods of 420-650 s while later Ugarte-Urra et al. (2004b) reported oscillations with periods ranging from $600 \mathrm{~s}$ to $1100 \mathrm{~s}$. These oscillations were interpreted as an indicator of global magnetic-acoustic modes of the closed magnetic structures associated with BPs. Although oscillations with periods of a few minutes are more commonly known, longer periods were also found by Tian et al. (2008). The reason for these oscillations is still under debate. Parker (1988) suggested they are repetitive small-scale flares, i.e. micro-flares. Tian et al. (2008) suggested magneto-acoustic waves and/or a recurrence of magnetic reconnection. Ugarte-Urra et al. (2004b) found that there is a one-to-one relation between the magnetic flux in the bipole and the EIT $195 \AA$ coronal emission during the growing and decaying phase for the two BPs studied. The authors concluded that their results give further support to the idea that magnetic reconnection involving the interaction of two magnetic polarities is the more likely operating mechanism.

Regarding magnetic fields, BPs are found to be associated with small bipolar regions (Krieger et al. 1971; Golub et al. 1977; Webb et al. 1993; Brown et al. 2001). With the Big Bear Solar Observatory (BBSO) magnetograms, Webb et al. (1993) investigated the correspondence of evolving magnetic features of 25 X-ray BPs. They found 22 BPs associated with converging of the magnetic features, while 18 were associated with cancelling magnetic features. They also found the BPs were more likely associated with pre-existing magnetic features rather than newly emerging ones. From higher resolution observations during the Yohkoh mission, Harvey et al. (1994) further demonstrated that most BPs were associated with magnetic cancellation. Preś \& Phillips (1999) found that the time evolution of several BPs observed in EIT $195 \AA$ images was strongly correlated with the variation of magnetic flux as determined from MDI photospheric magnetograms. By studying a BP observed in EIT $195 \AA$ together with its MDI photospheric magnetograms, Madjarska et al. (2003) confirmed such a correlation. They also found that the BP existed until the complete cancellation of one of the polarities from the corresponding bipolar region. Ugarte-Urra et al. (2004a) also observed a linear dependence between the EIT $195 \AA$ intensity flux from a BP and the total magnetic flux of a photospheric bipolar region. They found that the increase of the coronal emission associated with a BP was linked to the emergence of a new magnetic field and the disappearance of coronal emission was associated with the cancellation of one of the polarities. In one of the cases, the disappearance in EUV emission took place three to four hours before the full cancellation of the weakest polarity.

As reviewed above, BPs are associated with magnetic cancellation in photospheric magnetograms. What does actually happen during the cancellation process? Zwaan (1987) suggested a few possibilities. One way is a simple submergence, when a pre-existing loop descends into the convection zone. Another scenario requires magnetic reconnection either above or below the photosphere, which is named reconnection submergence. The reconnection submergence was discussed in detail by van Ballegooijen \& Martens (1989). They pointed out that a certain distance between the two opposite polarities was required for this type of cancellation to happen. From simultaneous measurements of the magnetic field in the photosphere and chromosphere Harvey et al. (1999) concluded that the magnetic flux "is retracting below the surface for most, if not all, of the cancellation sites studied". Furthermore, Priest et al. (1994) developed a model of BPs based on converging motions of magnetic features which can trigger magnetic reconnection and thereby energize a BP. The model was further developed in three-dimensions by Parnell et al. (1994) and tested by two-dimensional numerical experiments by von Rekowski et al. (2006a,b). In the model of Priest et al. (1994), (called Converging Flux Model), a certain interaction distance is also required to start the reconnection and then energize the BP. Evidence of this model was provided by Madjarska et al. (2003) who found that the BP appeared in EUV only when two opposite polarities were $10^{\prime \prime}$ apart. Possible evidence of magnetic reconnection in BPs was also confirmed by magnetic field reconfigurations (Pérez-Suárez et al. 2008; Alexander et al. 2011; Zhang et al. 2012).

X-ray jets are dynamic phenomena which represent collimated plasma flows from coronal BPs (see Paper II, III, Wang et al. 1998; Doschek et al. 2010). They were first discovered in soft X-rays by Shibata et al. (1992) and are mostly associated with mixed polarity regions (Shimojo et al. 1998). Magnetic reconnection is believed to be the main mechanism of their formation (Yokoyama \& Shibata 1995; Moreno-Insertis et al. 2008). A more detailed introduction on X-ray jets can be seen in Paper III and the references therein.

As a continuation of Papers I, II, and III and based on the brightenings identified in Paper II, we focus here on the evolution of magnetic flux associated with transient brightenings responsible for the small-scale evolution of coronal holes. A comparison with only a few events in the quiet Sun is also provided. Such a detailed study of the magnetic field of small-scale transients in the solar atmosphere is unprecedented thanks to the high-sensitivity, cadence and resolution of the SOT/Hinode data which has been pivotal for achieving the aim of our study. In Sect. 2, we describe the observations used in this study and the methods used in the data calibration and analysis. In Sect. 3, we 
Table 1. Description of the observations used in this study.

\begin{tabular}{lccccc}
\hline \hline Date & Observing period & \multicolumn{2}{c}{ Cadence $(\mathrm{s})$} & Observed & SOT FOV size \\
& $(\mathrm{UT})$ & XRT observations & SOT magnetograms & region & \\
\hline 2007-Nov-09 & $06: 38 \rightarrow 14: 59$ & 40 & 90 & Coronal hole & $261^{\prime \prime} \times 148^{\prime \prime}$ \\
2007-Nov-12 & $01: 21 \rightarrow 10: 57$ & 40 & 90 & Coronal hole & $261^{\prime \prime} \times 148^{\prime \prime}$ \\
2009-Jan-10 & $11: 30 \rightarrow 16: 59$ & 60 & 45 & Quiet Sun & $46^{\prime \prime} \times 148^{\prime \prime}$ \\
2009-Jan-13 & $11: 22 \rightarrow 17: 34$ & 60 & 45 & Quiet Sun & $46^{\prime \prime} \times 148^{\prime \prime}$ \\
\hline
\end{tabular}

present the results. Our discussion and conclusions are given in Sect. 4.

\section{Observations and analysis}

\subsection{Data and calibrations}

The observations used in this study were taken by XRT (X-Ray Telescope, Golub et al. 2007) and SOT (Solar Optical Telescope, Tsuneta et al. 2008) onboard Hinode. They include four datasets. Two of them were taken in November 2007 pointing at an equatorial coronal hole, and another two were taken in January 2009 in quiet-Sun regions. In Table 1 we list all details of the observations.

The XRT observations were taken with the Al_Poly filter whose temperature response function peaks around $8 \times 10^{6} \mathrm{~K}$. The pixel size of the X-ray images is $1^{\prime \prime} \times 1^{\prime \prime}$. The standard procedure (xrt_prep.pro) from the solar software (SSW) was used for the data reduction. The observations were carefully examined and any frames which were seriously affected by cosmic rays or instrumental effects were then excluded. In addition to the jitter correction, the images were further cross-correlated for the removal of the residual jitter.

The SOT observations used in this study include two types of data, series of Stokes $V$ and $I$ polarimetric images taken with the Narrowband Filter Imager (NFI) and Stokes $I, Q, U, V$ polarimetric spectra obtained with the Spectro-polarimeter (SP). The NFI filtergrams (FG) $V$ and $I$ images were taken in the Na I $5896 \AA$ spectral line which measures the magnetic field in the chromosphere (Ichimoto et al. 2008). The pixel size of the magnetograms is $0.16^{\prime \prime} \times 0.16^{\prime \prime}$. Each SP scan took about $20 \mathrm{~min}$ to obtain. The SP images have a pixel size of $0.32^{\prime \prime} \times 0.32^{\prime \prime}$. The standard softwares available from SSW (i.e. $f g \_$prep.pro and $s p \_$prep.pro) were used to calibrate the data. Some of the $V / I$ magnetograms which were badly affected by cosmic rays were not included. The time series of Stokes $V / I$ magnetograms were further corrected for the jitter effect via a cross-correlation method. After this correction a region of 50 pixels (i.e. $8^{\prime \prime}$ ) at each edge of the original field-of-view was discarded.

To convert the $V / I$ values into physical units, we adopted a method which was applied by Chae et al. (2007) to Fe I $6302.5 \AA$. In this method, the magnetic strength $(B)$ is assumed to be linear to $V / I$, i.e. $B=\beta \frac{V}{I}$. Although Chae et al. (2007) mentioned that $\beta$ would vary with magnetic strength, they also suggested that it is a good approximation to assume it as a constant in a region with similar solar activity. To obtain the best linear fit, we used a slightly different formula, $B=\beta \frac{V}{I}+B_{0}$, where $\beta$ and $B_{0}$ are constants. We produced the longitudinal magnetogram from the SP scan with the standard method instructed by the SOT analysis guide. We then averaged the FG V/I images obtained during this scan. Next, we re-binned the FG data to the same spatial scale as the SP magnetogram and co-aligned them. Finally, we applied a linear fit to the distribution of the SP longitudinal magnetic strength versus the FG $V / I$ values to get the calibration parameters of $\beta$ and $B_{0}$. The resulting values of the magnetic field can carry an error of up to $30 \%$ from the assumed radial field due to the viewing geometry. To correct the line-of-sight effect, the longitudinal magnetograms are then divided by cosine of the offset to the solar disk centre angle at each pixel (for details see Hagenaar 2001).

In order to co-align the X-ray images with the chromospheric magnetograms, TRACE $171 \AA$ and $1700 \AA$ observations were used as intermediary. First we calibrated the TRACE data with trace_prep.pro which automatically aligns the observations of the two TRACE channels. We then co-aligned the X-ray images with the TRACE $171 \AA$ images and the SOT magnetograms with the TRACE $1700 \AA$ data. EIS He II $256.32 \AA$ and Fe XII 195.12 $\AA$ observations were also used to check the goodness of the co-alignment.

\subsection{Analysis methods}

After the data calibration and alignment, the brightening events identified in Paper II (in X-ray data) were overlayed on the SOT magnetograms. Further, a visual inspection was made to ensure which polarities are involved in an individual event. This was especially needed when a few events occurred close to each other. In Fig. 1 we show the X-ray images with each analysed event numbered and shown in dashed-line-boxed regions. The $\mathrm{X}$-ray images in this figure were generated by taking for each pixel the highest value obtained during the time intervals given in Table 1. We identified 22 events in the coronal hole datasets and six events in the quiet-Sun datasets as shown in Fig. 1.

To follow the evolving magnetic features, the feature tracking software SWAMIS (The Southwest Automatic Magnetic Identification Suite, DeForest et al. 2007; Lamb et al. 2008, 2010) was used in the present study ${ }^{1}$. The tracking procedures include feature detection, feature identification, feature association, tabulation and event classification. The details on the procedures and a user guide of the software can be found on the website. Gaps in the datasets can seriously mislead the tracking program, therefore, a linear interpolation was applied to fill these gaps. In SWAMIS, a low and a high threshold have to be assigned for the feature detection. Due to different fields-of-view, the high thresholds were not uniform for all datasets, thus five sigma of the magnetic field strength of all pixels from each dataset was used for the high threshold. In order to detect weak magnetic features, in each dataset we randomly selected 100 pixels from magnetic features that we determined as "weak". The average magnetic strength of these pixels was determined to be $25 \mathrm{Mx} \mathrm{cm}^{-2}$. Thus we used $25 \mathrm{Mx} \mathrm{cm}^{-2}$ as the low threshold. The downhill method was used in feature identification. This

\footnotetext{
1 SWAMIS can be freely downloaded from http: //www . boulder. swri . edu/swamis
} 

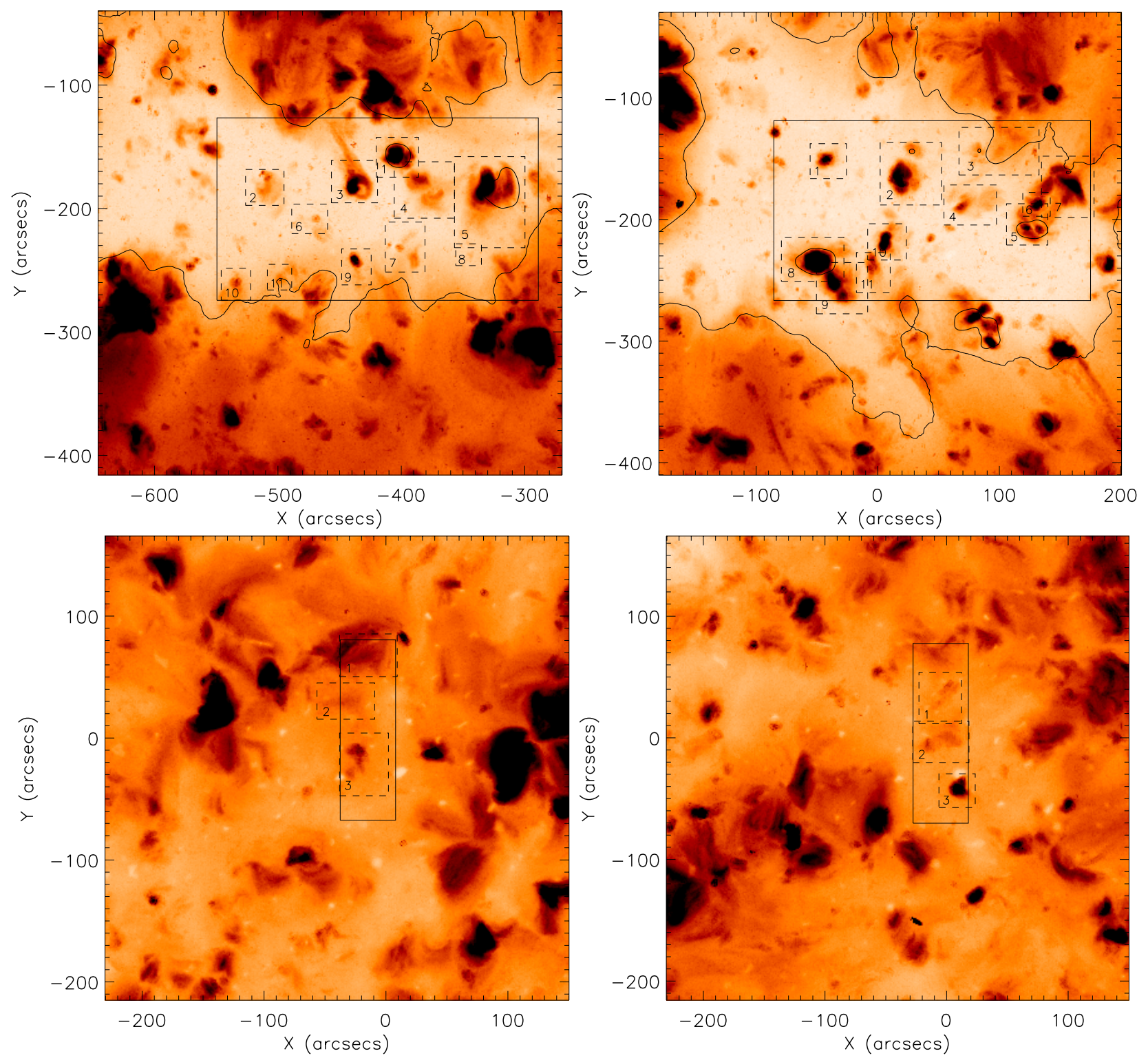

Fig. 1. Generated X-ray images (see the text in Sect. 2.2) of an equatorial CH on 2007 November 9 (top left) and November 12 (top right), the quiet Sun 2009 January 10 (bottom left) and January 13 (bottom right). The images are shown with an inverted colour table. The SOT/Hinode field-ofviews are marked as rectangles (solid lines). Features within the dashed-line boxes are the events studied in this paper. For the two $\mathrm{CH}$ datasets shown in the top panel, the black solid contour plot outlines the coronal hole boundary as seen at the beginning of each observing period.

method determines first a local maximum of magnetic flux density and then expands down towards zero gradient of flux density which is defined as the edge of one magnetic feature and its size. The magnetic feature is then followed until it disappears or merges with another feature, which determines its lifetime. The minimum size and lifetime of features to be detected was set to four pixels (i.e. $0.64^{\prime \prime} \times 0.64^{\prime \prime}$ ) and three frames (i.e. $270 \mathrm{~s}$ for the CHs data and $135 \mathrm{~s}$ for the QS data). A new magnetic feature can be born from splitting of the preexisted magnetic feature, or a flux emergence. SWAMIS defines a magnetic feature that was born associated with a recently-born opposite magnetic feature or with a growing opposite magnetic feature as flux emergence. In our online material, we marked all magnetic features on the magnetograms with symbols (flux emergence with squares, and the other new born magnetic features with asterisks). However, these definitions do not always work as expected because of the very complex behaviour of magnetic features. Feature tracking is a very complex procedure and no automatic program can deal with it precisely (DeForest et al. 2007, and priv. comm. with Parnell, C. E.), therefore, in detailed case studies it is useful to be complemented by visual analysis. The simultaneous X-ray images and magnetograms for each event were also reviewed by mean of naked eye. All important changes were noted and organised in tables (see Table A.1 as an example) $)^{2}$.

\section{Results}

\subsection{Magnetic field distribution in the coronal hole and the quiet Sun}

We present in Figs. 2 and 3 the full SOT field-of-view (FOV) images of the coronal hole and the quiet-Sun regions respectively.

\footnotetext{
2 All the movies, together with the notes are provided as online materials at http://www.arm.ac.uk/highlights/2012/603/bpmag . html
} 

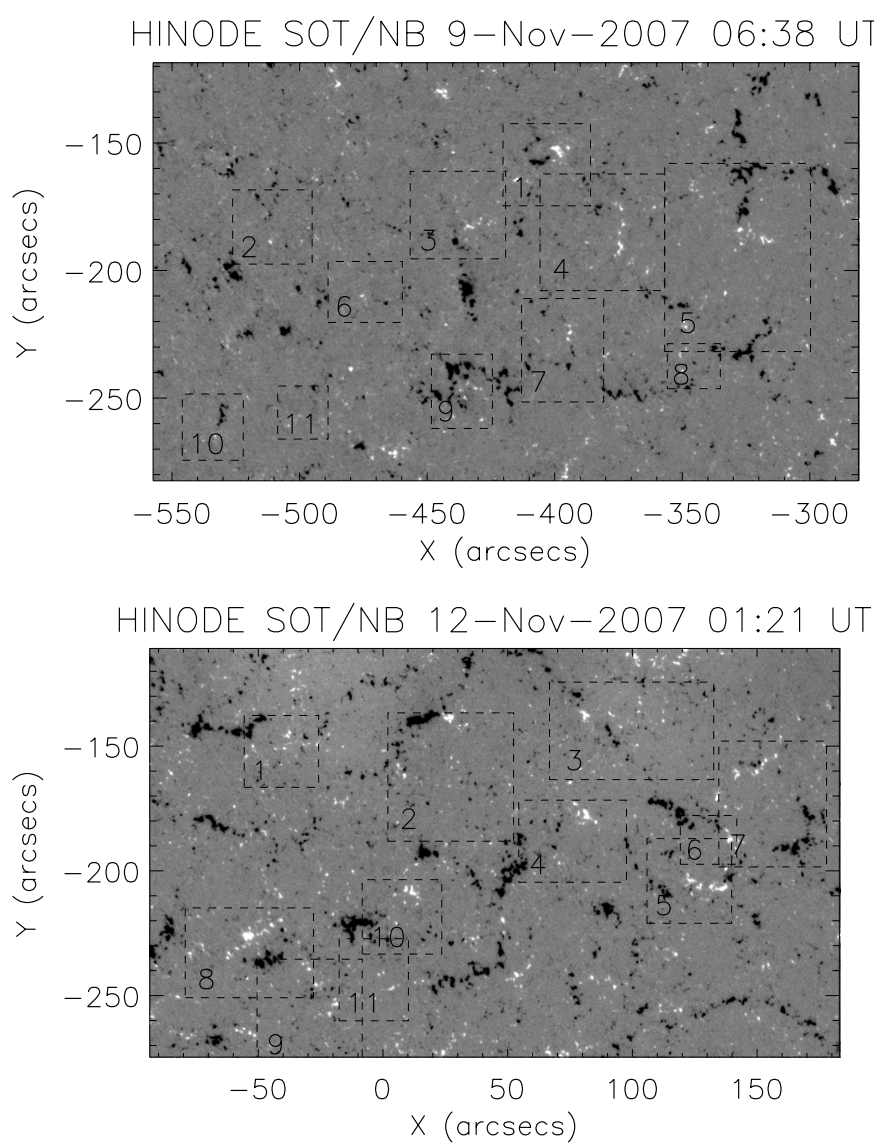

Fig. 2. Full field-of-view of the SOT longitudinal magnetograms of the coronal hole analysed in this study. Overplotted with dashed lines are the FOVs of the events identified in X-rays, which are shown in the top panels of Fig. 1.

A visual inspection of Fig. 2 reveals that the dominant flux in the coronal hole is of negative polarity which also forms the largest concentration of magnetic flux in terms of total flux. To compare the distribution of positive and negative fluxes in both regions, we calculated the average flux for each magnetic feature (see in Sect. 2.2 the definition of a magnetic feature) over its lifetime. In Fig. 4 we show the derived flux distribution for the four days of observations. In the $\mathrm{CH}$ the number of magnetic features with negative flux is more than four times higher with respect to the positive flux features. Both distributions peak at the same flux strength which is around $3-4 \times 10^{16} \mathrm{Mx}$, compared with $2 \times 10^{16} \mathrm{Mx}$ in the quiet Sun. In the quiet Sun the number of magnetic features is much more equivalent for both the negative and the positive flux. However, the limited fields-ofview of the quiet-Sun datasets can easily cause a small flux imbalance, especially on January 13. The fractional distribution of pixels with negative magnetic field can be found in Fig. 5. Again, the prevalence of a single polarity in the $\mathrm{CH}$ is very clear. The $\mathrm{CH}$ magnetic fields reach up to $600 \mathrm{Mx} \mathrm{cm}^{-2}$ while the quiet Sun contains magnetic field concentrations only up to $400 \mathrm{Mx} \mathrm{cm}^{-2}$. Almost $100 \%$ of the highest values of the magnetic field in the coronal hole are found in pixels occupied by the dominant polarity. It should also be noted that the fractional distribution of positive and negative polarities in the coronal hole did not change over a three day period which separates the two coronal hole datasets. A flux stronger than $100 \mathrm{Mx} \mathrm{cm}^{-2}$ remains imbalanced in the quiet-Sun regions because of the small fields-of-view.
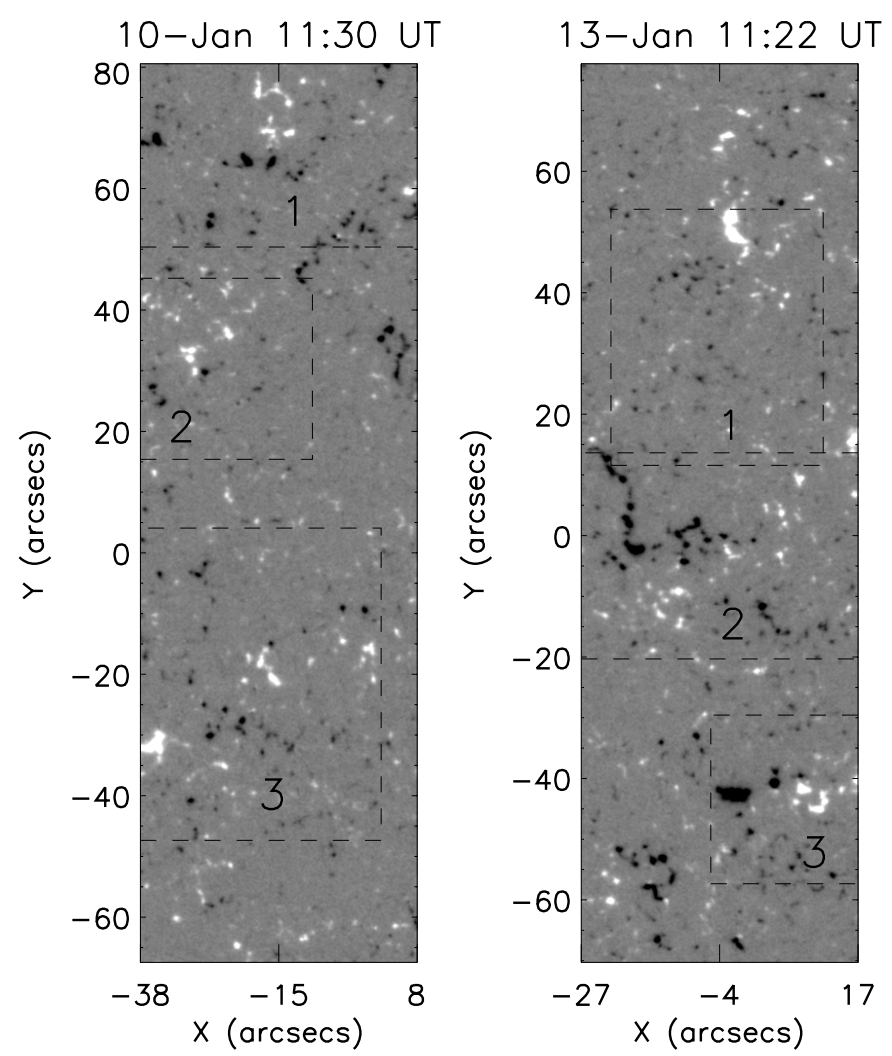

Fig. 3. Full field-of-view of the SOT longitudinal magnetograms of the quiet Sun analysed in this study. Overplotted with dashed lines are the FOVs of the events identified in X-rays, which are shown in the bottom panels of Fig. 1.

\subsection{X-ray intensity variations and magnetic field evolution of brightening events in coronal holes}

With SWAMIS, we were able to follow the magnetic features from their birth to death, studying their behaviour including emergence, convergence, splitting, mergence and cancellation. All events in the coronal hole resulted from the interaction of bipolar magnetic regions. From 22 brightening events, seven were seen in the X-ray images at the start of the observations while 12 appeared during the observations but their corresponding bipolar regions were already present at the beginning of the observations. Three events were formed during the observations together with the formation of their corresponding bipolar region. In most of the cases (19 out of 22), one of the polarities forms a "stable" centre (which can be a single large polarity or a group of closely scattered small polarities). The stable polarity remains in the same place and in only a few cases when the central polarity exists for more than a few hours, it may move a few arcseconds usually approaching the opposite polarity. The stable polarity tends to be the stronger polarity in the bipolar region. In eleven cases this is the negative polarity (i.e. dominant polarity in the coronal hole), in five cases it is the positive one, and in three cases it switches between polarities (on time scales of hours) depending on which one is the strongest at a particular time. The remaining three cases are inconclusive.

The evolution of the magnetic flux associated with a coronal transient brightening proceeds in a similar way for all events. All brightening events identified in the X-ray images are caused by magnetic flux emergence and a follow-up cancellation with the pre-existing and/or newly emerging magnetic flux. In some cases a magnetic feature (opposite to the stable magnetic feature 

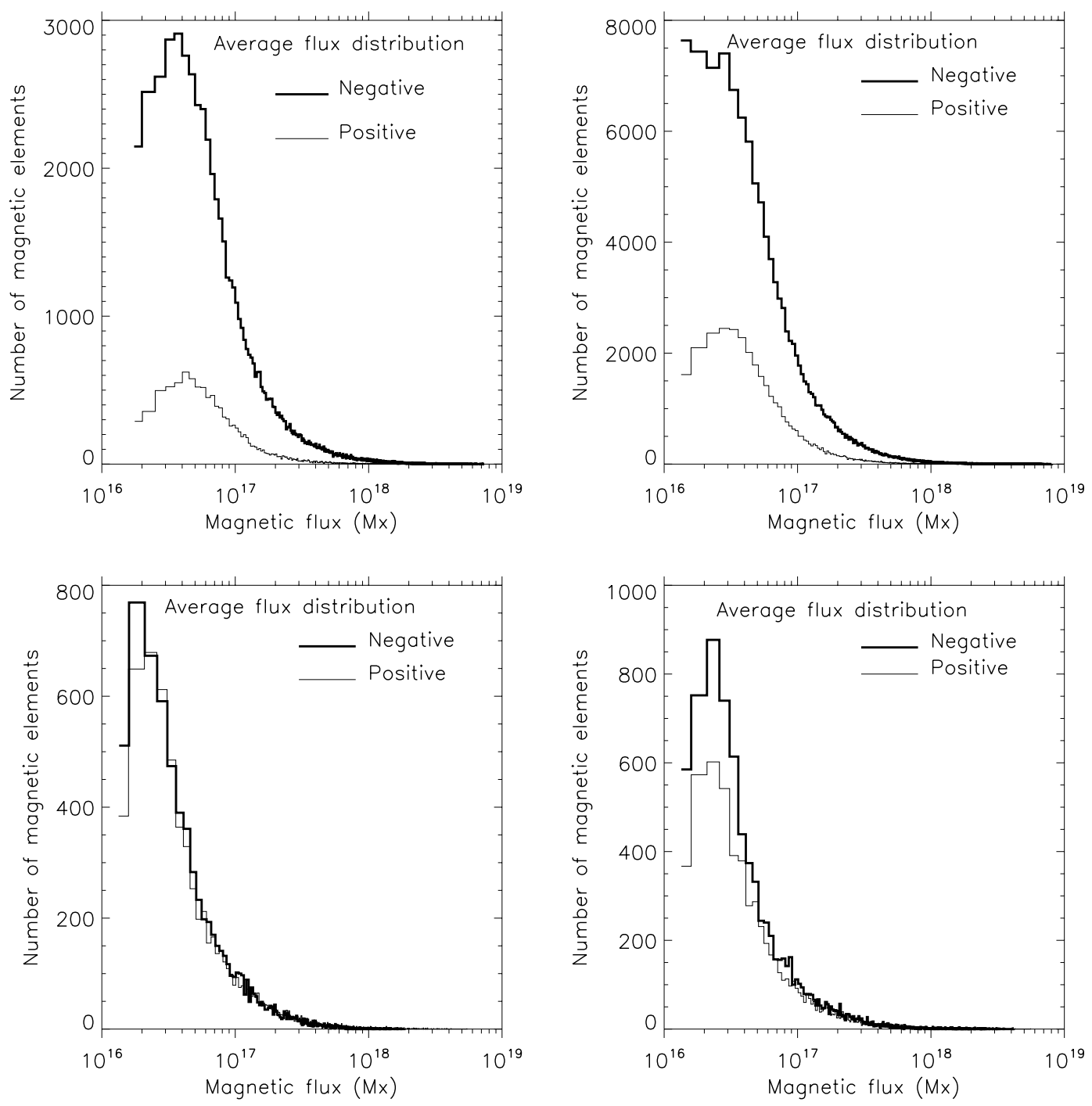

Fig. 4. Magnetic flux distribution of the CH (top left November 9, right November 12) and quiet-Sun (bottom left January 10, right January 13) magnetic features detected by SWAMIS.

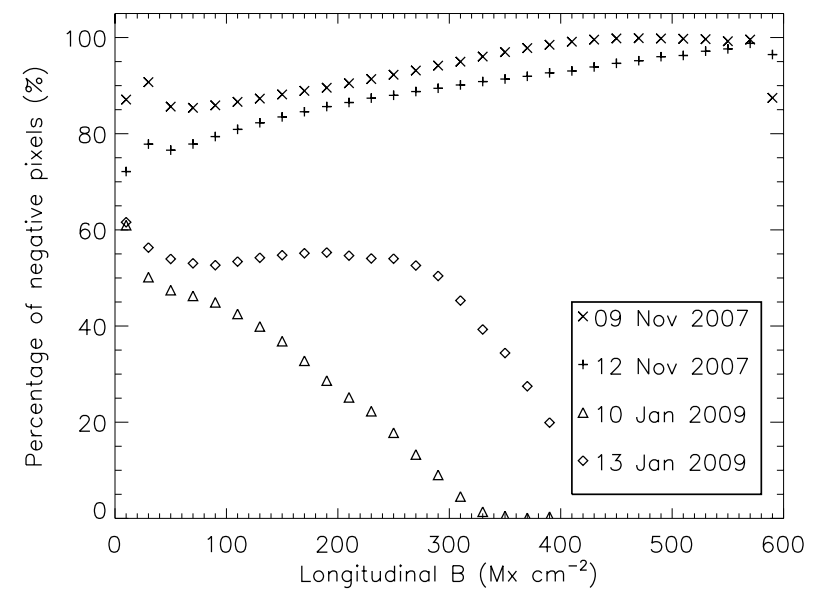

Fig. 5. Fractional number of pixels with negative longitudinal magnetic field for each day of the observations.

polarity) emerges close-by (a few arcseconds) from the stable polarity. The emergence is followed shortly after (a few tens of seconds) by cancellation. The emerging feature can also surface at a distance away (20" for instance) from the stable centre. The newly emerged polarity then starts moving towards the stable centre often becoming larger with time by merging with a feature of the same sign. Once the magnetic polarities get close to each other and start cancelling, a brightening will appear in the $\mathrm{X}$-ray image. However, from the visual analysis we established that there are many more magnetic cancellation sites than X-ray brightening events. We compared the magnetic flux (unsigned total) involved in magnetic cancellation while a brightening was observed in X-rays with the unsigned total magnetic flux when no X-ray brightening was present. We found that the magnetic flux involved in brightening events is two times larger than the cases without an X-ray response. We speculate that there is a threshold of the amount of magnetic flux involved in cancellation above which brightening would occur at X-ray temperatures. This subject, however, needs further detailed investigation which will be tackled in future work. Magnetic flux emergence is also important in maintaining the field strength of a magnetic feature during its cancellation. Flux cancellation and emergence are often seen to happen simultaneously at the same location.

The X-ray/magnetic-flux correlation analysis demonstrates a strong correspondence of the magnetic positive and/or negative 


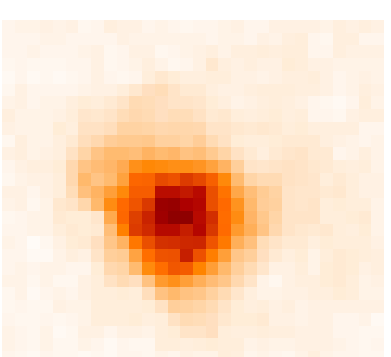

06:38:09 UT

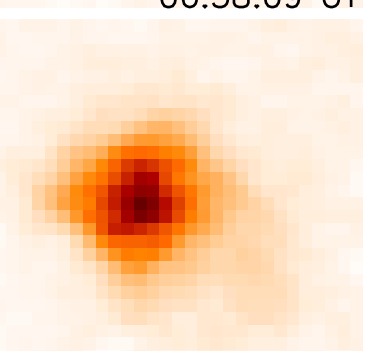

07:21:10 UT

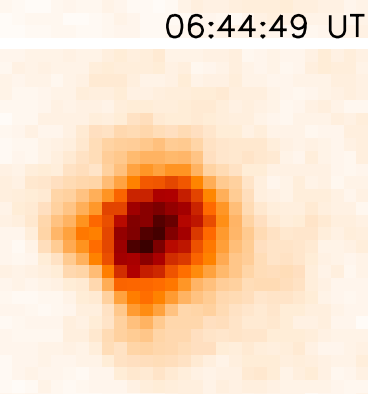

07:26:30 UT

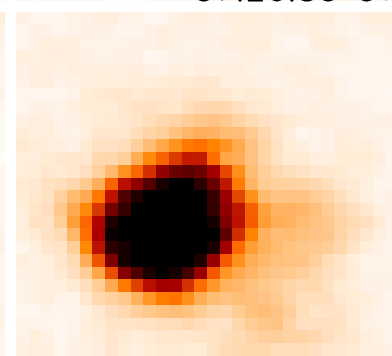

09:44:17 UT

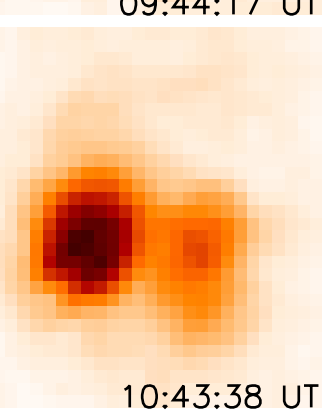

09:16:36 UT
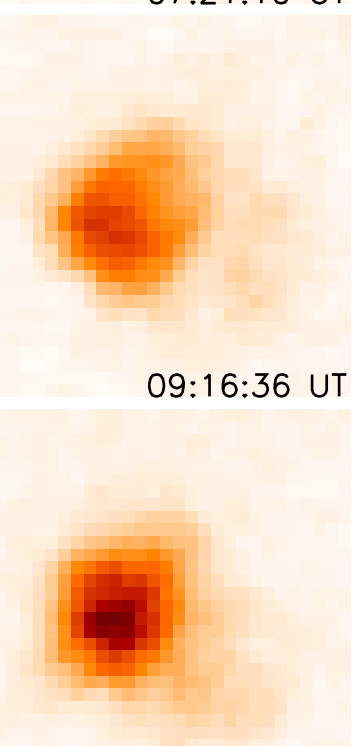

10:27:57 UT
10:43:38 UT

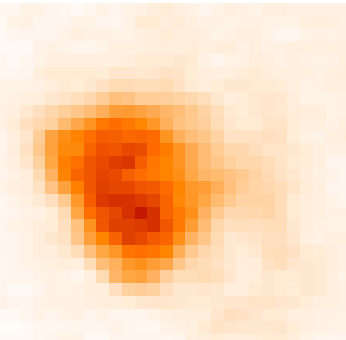

06:59:30 UT

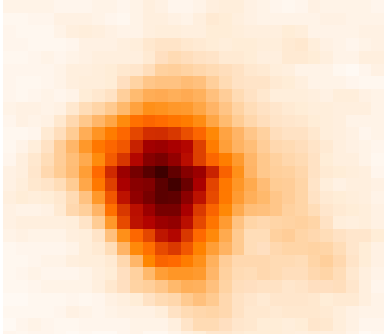

07:07:50 UT

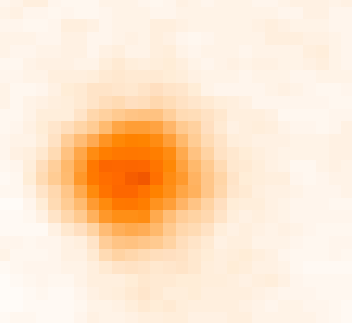

07:53:34 UT
08:17:15 UT

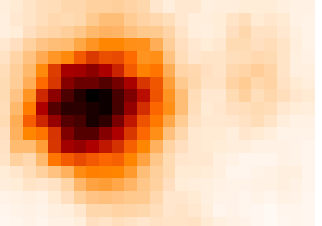

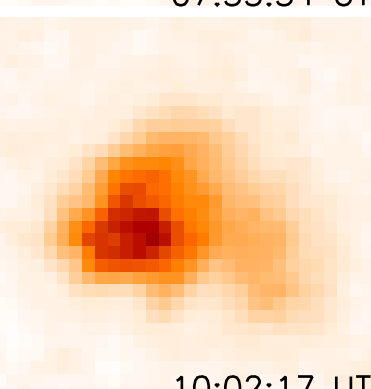

10:02:17 UT

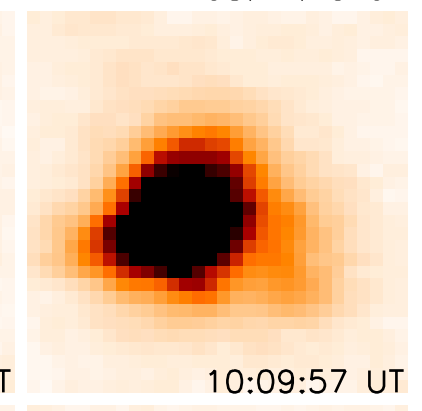

12:55:30 UT

14:52:16 UT

Fig. 6. Temporal evolution of a $\mathrm{CH}$ bright point seen in X-ray on November 9 (event No. 1). The images are displayed in reversed colour table. The field-of-view size is $30^{\prime \prime} \times 30^{\prime \prime}$. An animated version is available online, cf. Fig. A.1.

fluxes with the long-term (in the order of a few hours) changes in the X-ray emission (see the discussion in the following subsection for more details). However, in some cases even changes as short as one hour can be observed (see the X-ray and positive flux variations in Fig. 11 for example). The radiance variations of isolated bright points in the coronal hole (five cases) correlated with the magnetic flux changes in both polarities. In $50 \%$ of the cases (eleven) the radiance variations correlate ONLY with the positive flux, i.e. the subordinate polarity in the $\mathrm{CH}$. In five out of 22 cases the correlation in time follows either one or the other polarity. In one case a very strong jet was followed in X-rays together with the emergence, evolution and submergence of the magnetic features associated with it.

Thanks to the very simple magnetic field configuration of the coronal hole (and in general any coronal hole) the evolution of each individual phenomenon can easily be analysed. In the following subsections, we give a detailed and further description of three examples (two $\mathrm{CH}$ bright points and one X-ray jet).
Note that in the online material we give the same full description (figures, movies and tables) for each analysed event.

\subsubsection{Magnetic evolution of two $\mathrm{CH}$ bright points}

In Fig. 6 we give an example of the temporal evolution of a $\mathrm{CH}$ bright point on November 9 (event No. 1) as seen in X-rays. The temporal evolution of the magnetic polarities associated with this event is presented in Fig. 7. The corresponding lightcurves in $\mathrm{X}$-rays, positive and negative magnetic flux are shown in Fig. 8. A detailed description of the BP evolution is given in Table A.1. The intensity variation of the BP is apparent with peaks of the X-ray emission at 06:44 UT, 07:07 UT, 07:26 UT, 08:17 UT, 09:44 UT, 10:10 UT, 10:43 UT and 14:52 UT, with an overall periodicity of $\sim 20 \mathrm{~min}$. The shape and projected size of the BP change in time as well. We give an example of an emerging magnetic feature (denoted by a white arrow in Fig. 7) which moves 

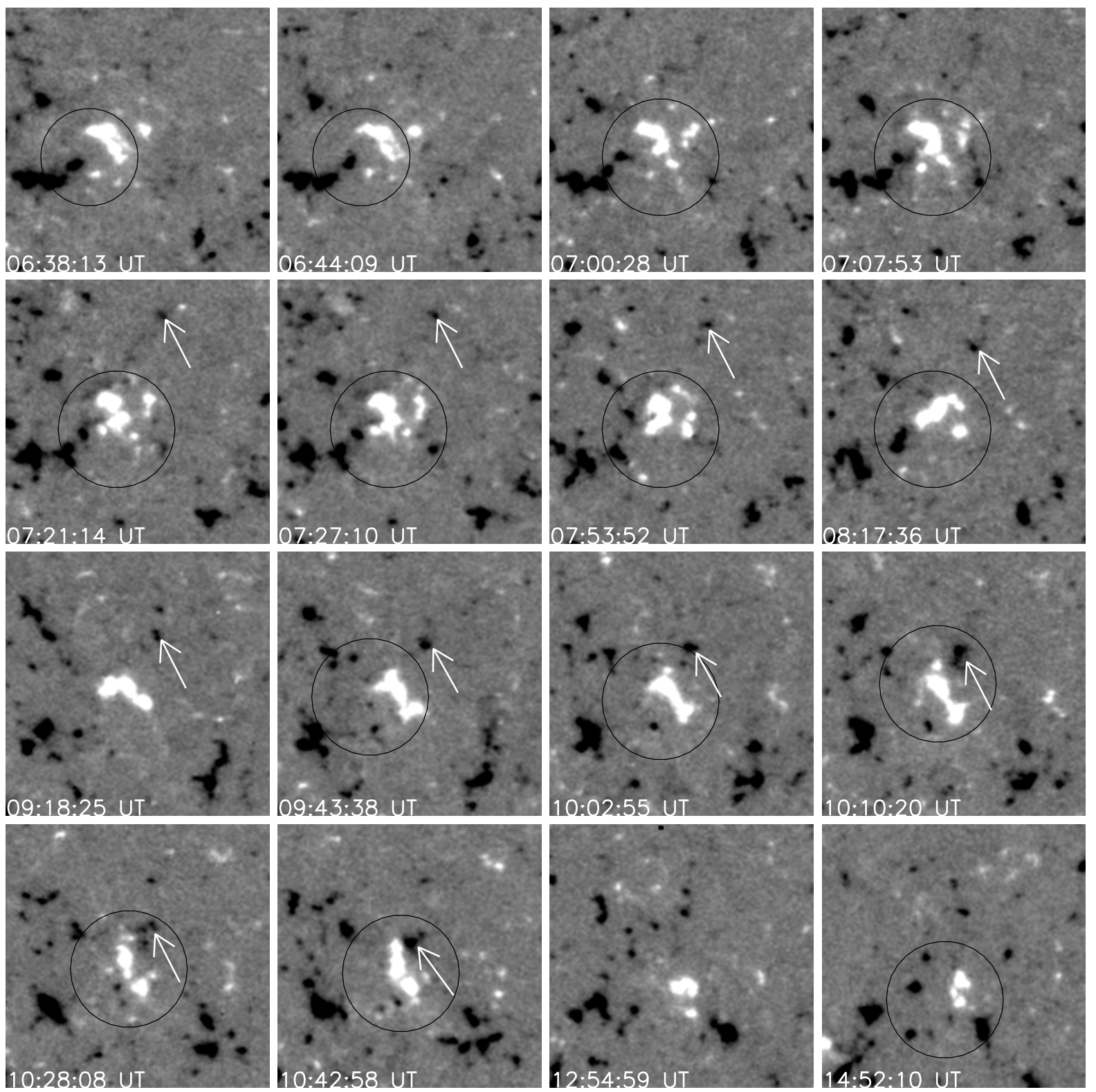

Fig. 7. Longitudinal magnetic field images of the $\mathrm{CH}$ bright point shown in Fig. 6. The field of view is the same as in Fig. 6, and the images are scaled from $-70 \mathrm{Mx} \mathrm{cm}^{-2}$ to $+70 \mathrm{Mx} \mathrm{cm}^{-2}$. Whenever the magnetic cancellations are observed, the site is outlined by a black circle on the corresponding image. The circle is adjusted as precisely as possible to include only the involved magnetic features. A white arrow points at an emerging magnetic feature. The field-of-view has a size of $30^{\prime \prime} \times 30^{\prime \prime}$. An animated version is available online, cf. Fig. A.1.

towards the pre-existed opposite polarity, causing magnetic cancellation. The magnetic feature emerges at 07:21 UT and affects the event around 10:10 UT which results in a strong X-ray emission increase in this event (Fig. 6 at 10:09:57 UT, and in Fig. 8, the highest peak in the lightcurve).

Radiance oscillations in BPs have been the subject of several studies in the past (see Sect. 1). They were interpreted as the signature of propagating waves though suggestions were also made that these emission spikes can be due to repetitive magnetic reconnection. The lightcurves of the negative and positive magnetic fluxes derived here show similar fluctuations. The question is what is the origin of these small-scale variations of the magnetic flux? The lightcurves were smoothed with five data points to remove the short period variations. The five data points here correspond to $7.5 \mathrm{~min}$ (the cadence of our data is $1.5 \mathrm{~min}$ ). A visual inspection of event No. 1 on November 9 and all other events suggests that the magnetic flux lightcurve variations are mostly due to emerging and cancelling magnetic features.

Event No. 2 on November 12 best describes the magneticflux/X-ray-emission correlation. In Fig. 9 we show the X-ray image sequence and in Fig. 10 the magnetogram sequence. The corresponding lightcurves are given in Fig. 11. The event first appeared in X-rays at 04:23 UT when a bipolar region was formed. The emergence of the positive magnetic features in this bipolar region started at the beginning of the observations (i.e. 01:21 UT). They emerged near a negative polarity and moved to another negative polarity to form the bipolar region. The negative polarity is the stable one and was formed from both preexisting and newly emerging magnetic flux. The X-ray emission variations of the event have a period close to an hour. The positive flux strongly correlates with the X-ray emission (Fig. 12). The recurring positive magnetic flux cancellations are associated 

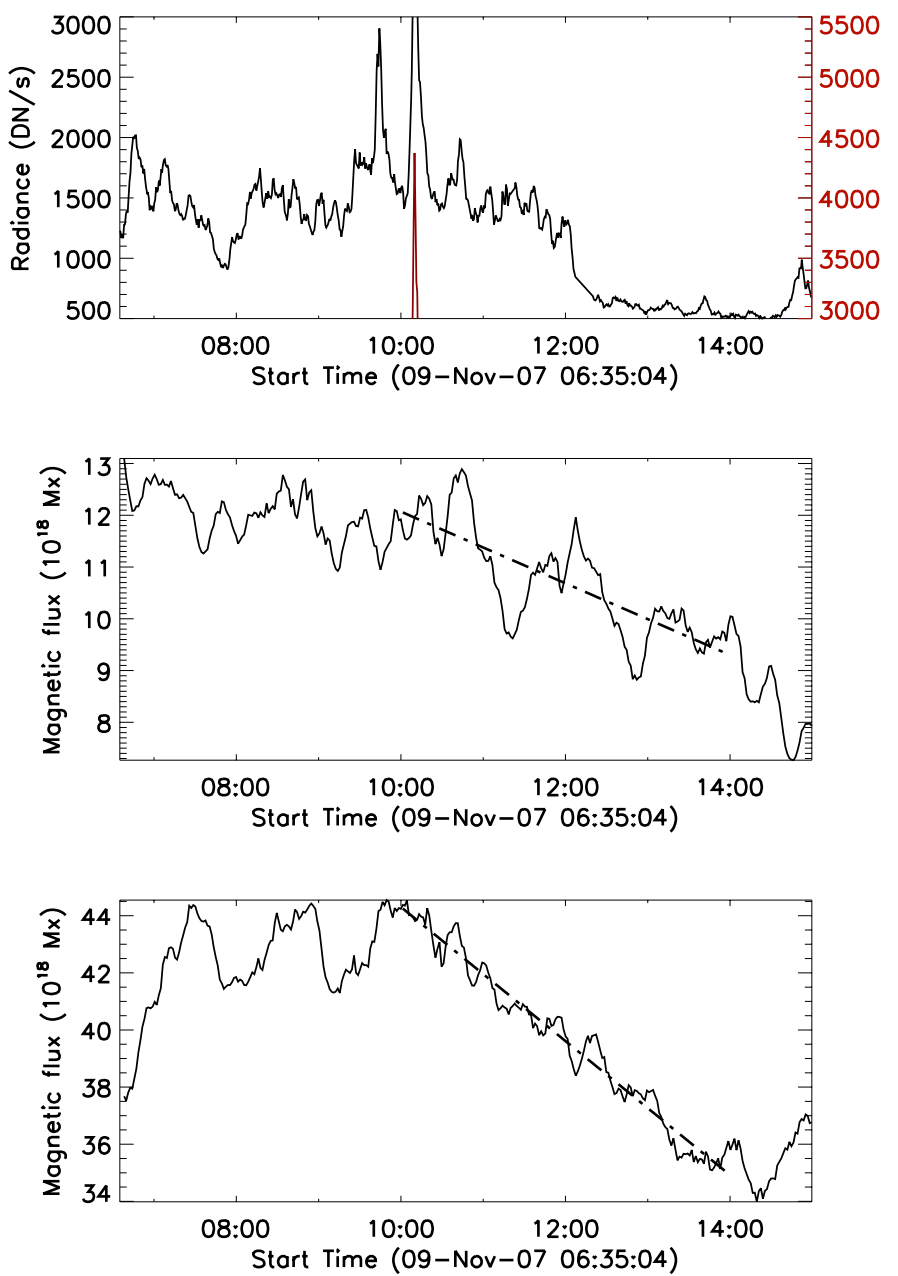

Fig. 8. Lightcurves (top: X-ray radiance; middle: positive magnetic flux; bottom: negative magnetic flux) of a $\mathrm{CH}$ bright point that is marked as No. 1 event on November 9 in Fig. 1. The lightcurves are extracted from the FOV shown in Figs. 6, and 7. The time $(x)$ axis is shown in unit of UT. For better scaling the peak of the X-ray lightcurve is cut off and resumed from bottom in red. The dash-doted-lines in the middle and bottom panels are linear fits of the corresponding magnetic flux from 10:00 UT to 14:00 UT.

with an intensity variation and can be followed in the online material (Fig. A.2). An example of an emergence and then cancellation is denoted by an arrow in Fig. 10. After emergence several small positive flux concentrations merge together forming larger flux concentrations. The cancellation with the negative magnetic features starts at around 06:21 UT and the positive polarity disappears around 10:00 UT. This typical process can be summarised as: a single polarity emerging away from an opposite polarity magnetic feature $\rightarrow$ becomes stronger and moves away from the emerging site $\rightarrow$ divides into a few features, each of them becomes stronger and/or merges with other features of the same sign $\rightarrow$ moves towards the opposite sign flux and cancels with it.

\subsubsection{Magnetic flux evolution of an X-ray jet}

In our observations, an X-ray jet was observed in the $\mathrm{CH}$ on 2007 November 9 (marked as No. 3 event in Fig. 1). The evolution of the jet in X-rays and its corresponding magnetic flux are shown in Figs. 13 and 14. The X-ray and magnetic flux lightcurves are given in Fig. 15. At the beginning of the observations (06:38 UT) the region is very quiet, i.e. no X-ray emission variations are observed. Around 07:17 UT a bright point emerges, showing fluctuations in its X-ray emission until around 11:30 UT. At 11:44 UT a brightening in a few pixels is followed by growth of the bright point. At 11:55 UT, a loop structure, which could be the site projection of a sigmoid, is formed and starts rapidly expanding producing a jet around 11:58 UT. When the jet first forms, the brightening system (the loop and the jet) has an inverted-Y-shaped. Both the jet and the loop then become brighter. Around 12:01 UT, the loop appears O-shaped. Around 12:02 UT, the jet evolves into two bright branches. The brightening system reaches peak emission around 12:05 UT, and then becomes weaker, returning to a single branch again around 12:07 UT. The jet disappears fully before 12:20 UT. Because of a data-gap between 12:08 UT and 12:20 UT, we could not follow the event during its weakening stage. A small bright point is still present when the observations resume at 12:20 UT. At 12:36 UT the bright point cannot be seen in X-rays. It flares again at 13:10 UT, and produces a small short-lived (about two minutes) jet around 13:21 UT. Around 13:24 UT, a small bright point remains but is weak. The brightening system completely disappears in X-rays after 13:30 UT.

Figure 15 shows the variation of the magnetic flux in the footpoints of the jet. As often seen in bright points, the magnetic flux at the footpoints of the jet clearly decreases after its eruption. Figure 14 displays the longitudinal magnetograms evolution of the region. At 06:38 UT, a bipolar region is already present. Around 07:17 UT when the bright point emerges, positive features are moving closer to the negative features and some weak features disappear due to cancellation. During the bright point intensity fluctuations, the positive features continue moving towards the negative ones. The cancellation of the negative flux shown in the boxed region in Fig. 14 starts shortly before 09:00 UT. After 09:00 UT the positive flux has also started to cancel, though additional positive flux emergence takes place before 10:00 UT. It is followed by a rapid flux cancellation starting at 10:40 UT. Around 11:40 UT, positive features are touching the negative ones. At this time the negative fragments are closely grouped together. Around 11:55 UT when the BP evolves into a sigmoid-like feature, a positive feature moves into the space between the two separate negative ones. When the jet starts (at around 11:58 UT), the magnetogram shows that the positive feature between two negative ones has become stronger and then starts to decrease. The positive feature is very small and weak at 12:20 UT, it disappears at 12:37 UT. After the major jet, both negative and positive features were more scattered and weaker, but the magnetic cancellation in the region continues. At around 12:22 UT, a small positive feature emerges, and it is highlighted by a white arrow after 12:37 UT in Fig. 14. The emerged feature becomes stronger and moves towards the negative one. It touches the negative feature and becomes smaller at around 13:21 UT when the small jet is formed. It disappears at the same time as the brightening system seen in X-rays fully fades away. After 13:30 UT, there are no positive features visible while the negative features in the region are much smaller than that at the beginning of the observation because of the cancellation.

\subsubsection{Magnetic flux cancellation rate of a BP and an X-ray jet}

We define the magnetic cancellation rate as $\frac{\Delta F}{\Delta T}$, where $\Delta F$ is the decrease of magnetic flux and $\Delta T$ is the time period of the decrease. In many cases the complex nature of the cancellation process does not allow us to calculate the cancellation rate. Fortunately, two events had a well isolated magnetic 


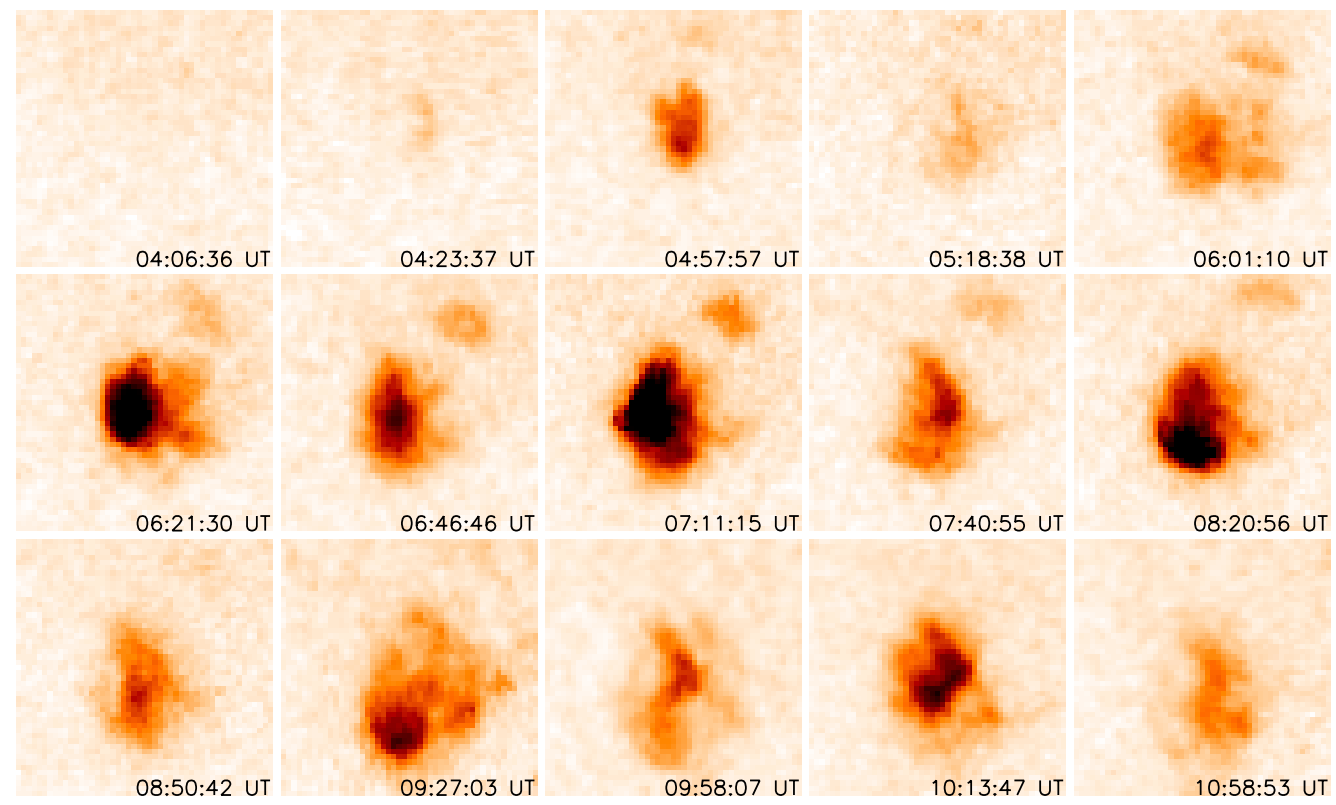

Fig. 9. As Fig. 6, but for a CH bright point observed on November 12 (marked as No. 2 event in Fig. 1). The field-of-view has a size of 50" $\times 50^{\prime \prime}$. An animated version is available online, cf. Fig. A.2.
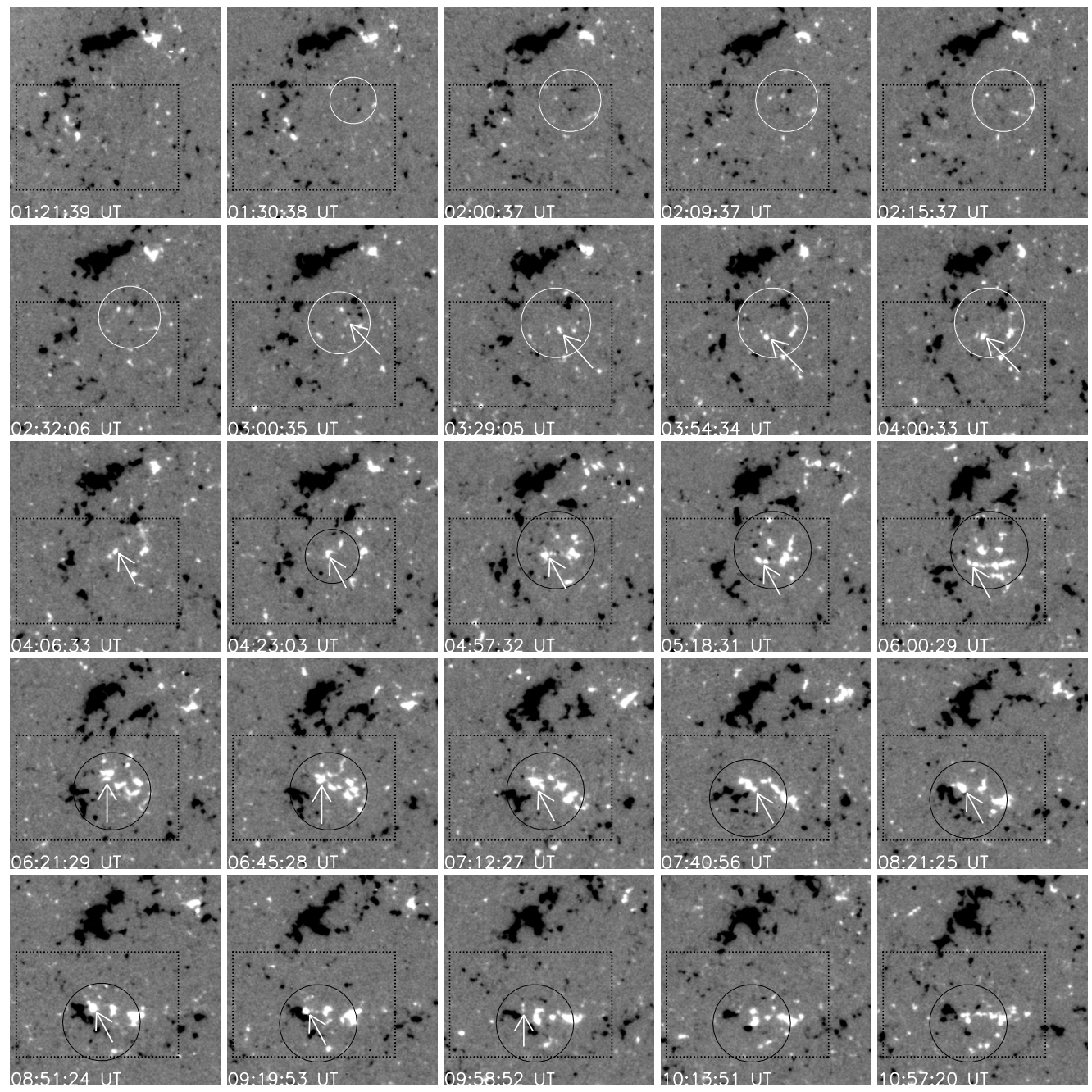

Fig. 10. As Fig. 7, but for the bright point shown in Fig. 9. The magnetograms are scaled from $-70 \mathrm{Mx} \mathrm{cm}^{-2}$ to $+70 \mathrm{Mx} \mathrm{cm}^{-2}$. The first two rows show the magnetic field evolution before the emergence of the brightening event. The white arrow points at an emerging magnetic feature which later cancels. The white circles emphasise a bipolar region created by magnetic flux emergence, while the black circles emphasise the area when and where magnetic cancellation occurs. Dotted lines outline the area where the magnetic flux lightcurve (Fig. 11) was extracted from. An animated version is available as online material, cf. Fig. A.2. 

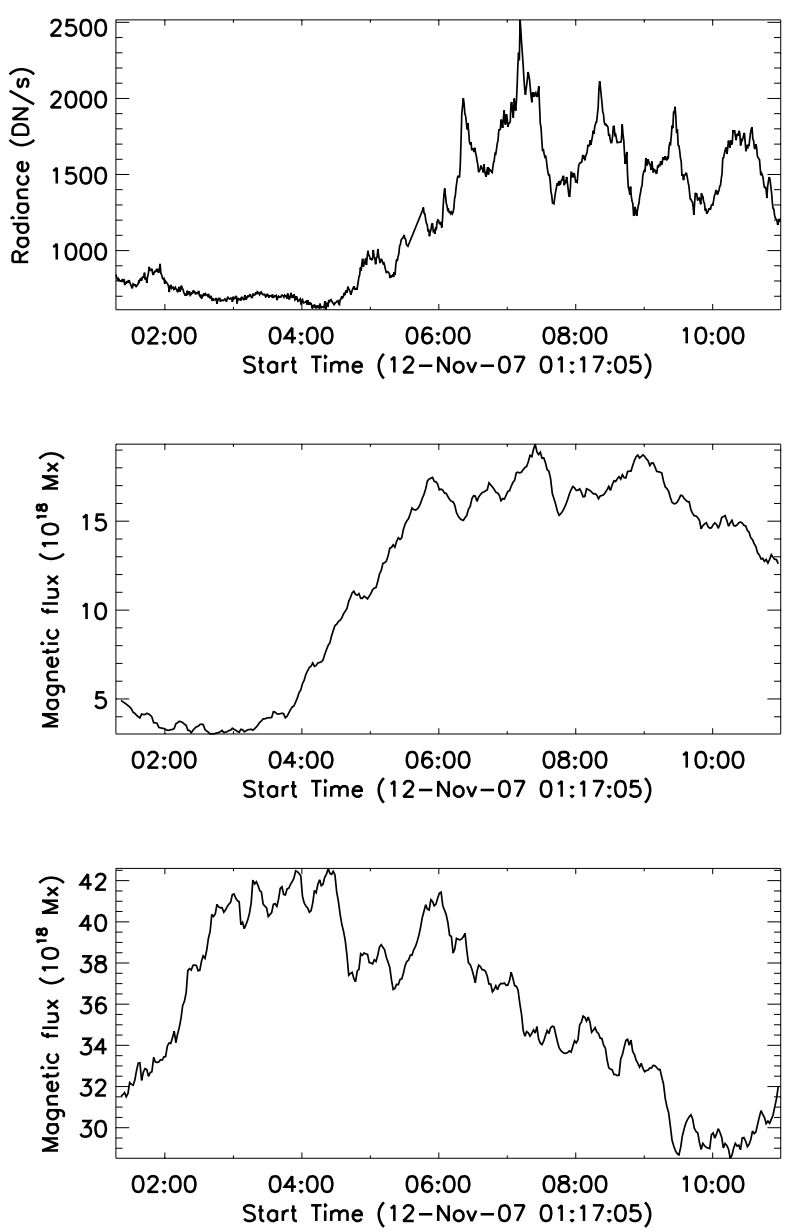

Fig. 11. As Fig. 8, lightcurves of a $\mathrm{CH}$ bright point that is marked as No. 2 event on November 12 in Fig. 1. The X-ray lightcurve is extracted from the FOV shown in Fig. 9 and the magnetic flux variations are extracted from the region outlined by dotted lines in Fig. 10.

configuration which permitted such an estimation. This is the decaying stage of event No. 1 (bright point) on 9 November (see Fig. 6) which was observed from 10:00 UT to 14:00 UT. During this time the magnetic flux lightcurves (see Fig. 8) show a decrease after the largest flaring (see the peak in the X-ray light curve displayed in red). The X-ray jet discussed in Sect. 3.2.2 also has a clear magnetic structure (see Fig. 14) and shows a very clear decrease of the magnetic flux lightcurves around and after the eruption (see Fig. 15 around 12:00 UT). In Figs. 8 and 15 , we overplot the corresponding linear fits on the corresponding unsigned magnetic flux of these two events. We obtained the magnetic cancellation rates from the linear fit as $1.9 \times 10^{14} \mathrm{Mx} / \mathrm{s}$ (positive polarity) and $6.5 \times 10^{14} \mathrm{Mx} / \mathrm{s}$ (negative polarity) for the bright point, and $3.0 \times 10^{14} \mathrm{Mx} / \mathrm{s}$ (positive polarity) and $7.9 \times 10^{14} \mathrm{Mx} / \mathrm{s}$ (negative polarity) for the X-ray jet. The flux cancellation rate of the positive polarity of the X-ray jet is about $60 \%$ higher than that of the bright point. The magnetic flux cancellation rate of the negative polarities (dominant one in this $\mathrm{CH}$ ) of the X-ray jet is only $20 \%$ higher with respect to the BP. The reasons why cancellation rates of negative polarities of two events are more equivalent need further investigation. A possible reason is that part of the flux included in the calculations is actually not involving to the events, which is very likely to happen for the dominant polarity (i.e. negative one in our cases).

As discussed previously, all brightening events are associated with magnetic cancellation. We presume that reconnection

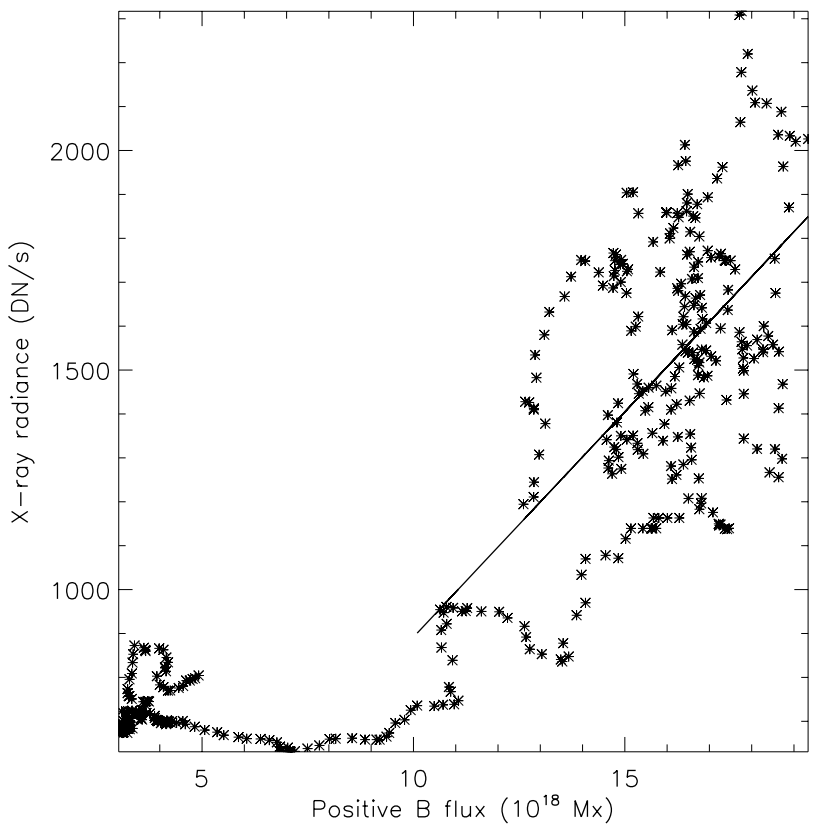

Fig. 12. Correlation analysis of the positive magnetic flux and the X-ray emission of event No. 2 on November 12.

of higher loops has produced short loops which consequently submerged and this is possibly what we see at photospheric levels as magnetic flux cancellation. We can only speculate that the higher rate of magnetic cancellation during the jet is a signature of a bursty physical process, e.g. magnetic reconnection, and the $\mathrm{X}$-ray jet formation is the signature of this process. The jet observation presented here is a single but very good example (unique concerning data cadence and resolution) of magnetic field evolution of an X-ray jet, however, drawing conclusions regarding how all jets exactly evolve is premature.

\subsection{X-ray intensity variations and magnetic field evolution of brightening events in the quiet Sun}

Events in the quiet-Sun regions studied in the present study are similar to those in the coronal holes, e.g. they are all associated with bipolar regions, magnetic cancellation is found to correlate with their behaviour in X-rays. From six events, three emerged before the beginning of the observations, and three emerged during the observing periods but their related bipolar regions can be tracked from the start of the dataset. In the quiet-Sun region magnetic features seed the whole region very densely and by moving frequently interact with each other in comparison to coronal holes. However, for the brightening events, a stable group of polarities is still found in three events. Brightening events in the quiet-Sun region also show fluctuation in their X-ray emission, and repeated magnetic cancellation is found to be associated with it. We presently studied only six events in the quiet-Sun regions. Due to the small sample, we can not make any general conclusion on quiet-Sun events. Observations of SDO/AIA/HMI and forthcoming IRIS will provide an opportunity to address this subject in a future study.

\section{Discussion and conclusion}

It is well known already for several decades that coronal holes form in unipolar photospheric magnetic fields. Thanks to the 


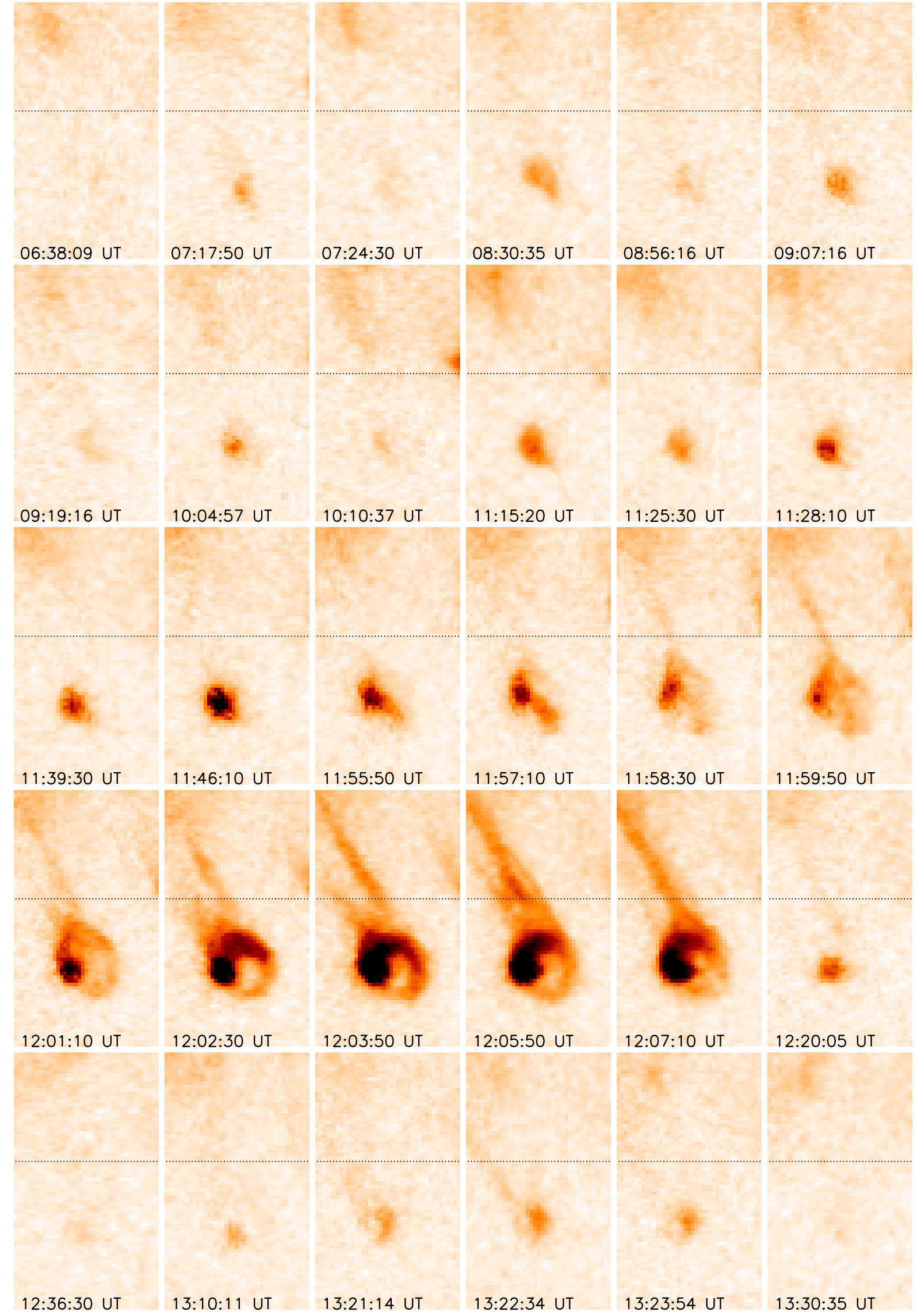

Fig. 13. As Fig. 6, but for an X-ray jet in a CH on November 9 (marked as No. 3 event in Fig. 1). The field-of-view has a size of $43^{\prime \prime} \times 75^{\prime \prime}$. The corresponding magnetograms of the area below the black dotted line are shown in Fig. 14. An animated version is available online, cf. Fig. A.3. 

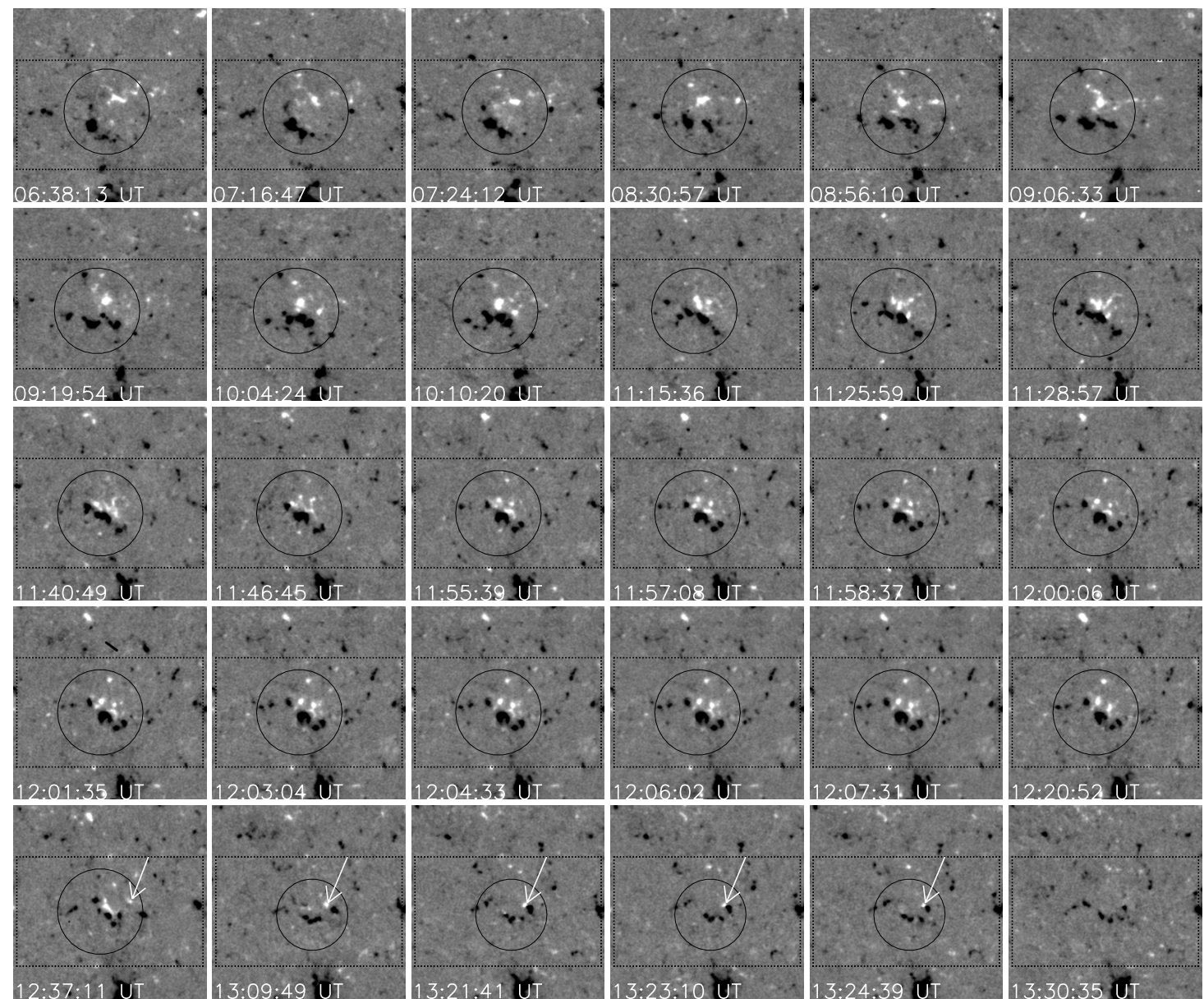

Fig. 14. Longitudinal magnetic field images of the X-ray jet shown in Fig. 13. The field-of-view corresponds to the area below the dotted lines in

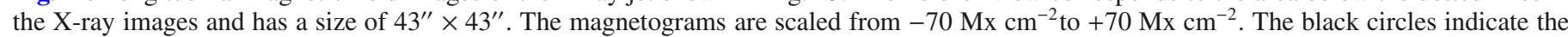
magnetic cancellation sites. The white arrow points at an emerging positive magnetic feature. The black dotted lines outline the region where the magnetic flux lightcurves were extracted from. An animated version is available online, cf. Fig. A.3.

high-resolution longitudinal magnetic field observations provided by SOT/Hinode, we were able to follow the magnetic field evolution of quiet-Sun and coronal-hole regions at high cadence and small scale. We investigated the role of small-scale transients in the evolution of the magnetic field in an equatorial coronal hole. We used the magnetic feature tracking procedure SWAMIS to follow magnetic feature emergence, movement, mergence, coalescence, cancellation etc. In addition, a visual analysis of each individual phenomenon simultaneously recorded by XRT and SOT was carried out.

We found that in the coronal hole the number of magnetic features of the dominant polarity (in the present case, this is the negative polarity) is four times higher than the non-dominant one. The magnetic field concentrations in the coronal hole reach $600 \mathrm{Mx} \mathrm{cm}^{-2}$ with respect to the quiet Sun where no concentrations with a field strength of more than $400 \mathrm{Mx} \mathrm{cm}^{-2}$ were detected. Ito et al. (2010) found more kilo-Gauss magnetic field concentrations in a polar coronal hole than in the quiet Sun. They explained the difference in the strength of the magnetic field of the two regions by the higher chance of collision, reconnection, magnetic energy loss and submergence of positive and negative features in the quiet Sun which would easily weaken the field strength. We believe that additional factors such as a different magnetic field diffusion and transportation in comparison to the quiet Sun as well as sub-photospheric processes may play a very important role. This will be a subject for future study.
Zhang et al. (2006) analysed the magnetic flux distribution in a small coronal hole using high signal-to-noise level (2 G) magnetic observations from BBSO. They concluded that if one polarity dominates the network field, the opposite one will dominate the intranetwork field (i.e. weak field). In this study, we also found that the dominant polarity in the coronal hole forms large concentrations of magnetic field while the opposite polarity is mostly dispersed in the form of weaker field concentrations. The magnetic flux distribution in the intranetwork region is not conclusive because its nature of weak field is a big challenge to the sensitivity and signal-to-noise of the instruments. We noticed that the supergranulation configuration appear to have preserved its general shape during approximately nine hours of observations though the large concentrations in the network (the dominant polarity which sustains the coronal hole open magnetic field) did evolve and/or were slightly displaced, and their strength either increased or decreased. All changes were caused by the interaction of the network field (mostly the dominant polarity) with the emerging opposite polarity magnetic field. We observe in 19 of 22 events in the coronal hole a single stable polarity which in eleven cases is the dominant polarity. That strongly suggests that the formation of small-scale transients is due to the interaction of pre-existing (often long-lived) magnetic flux with newly emerging opposite polarity flux.

Our results show that all brightening events are associated with bipolar regions. A bipolar region is not formed by only 

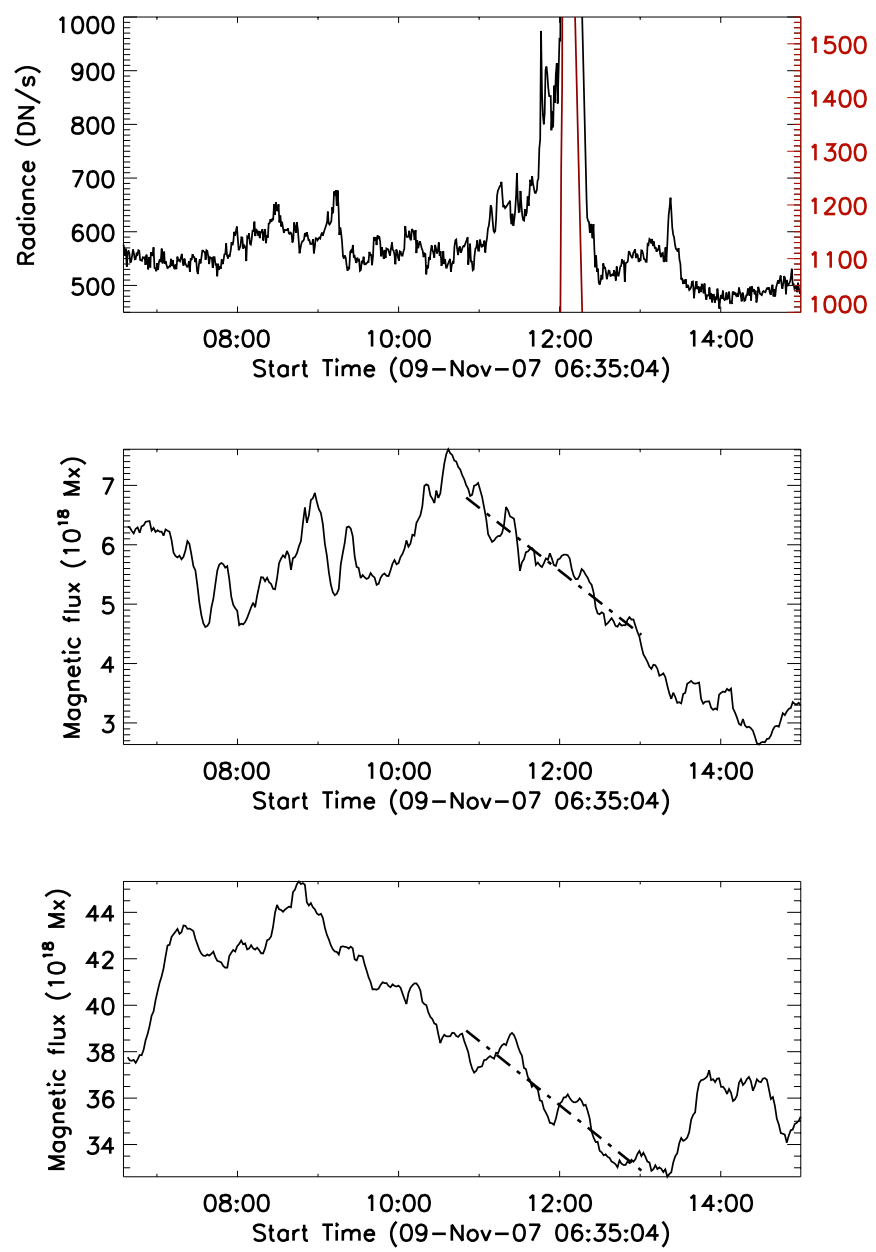

Fig. 15. Lightcurves of the X-ray jet marked as No. 3 event on November 9 in Fig. 1. The X-ray lightcurve (top) is extracted from the FOV shown in Fig. 13 and the magnetic flux lightcurves (positive - top and negative - bottom) are extracted from the region outlined by black dotted lines in Fig. 14. The time $(x)$ axis is shown in units of UT. The peak of the X-ray lightcurve (top) is cut off and resume from below in red. The dash-doted-lines in the middle and bottom panels are linear fits of the corresponding magnetic flux from 10:50 UT to 13:00 UT.

two simple opposite magnetic polarities but each polarity is organised in many magnetic features. From KPNO observations, Golub et al. (1977) found that bipolar regions did not exist in newly emerged and decayed X-ray bright points. Here, some of the studied events were followed from their emergence, through their entire lifetime until their full disappearance. Bipolar regions were found in the footpoints of the events before the event appearance at X-rays and were still present (although weak) after the decay of the feature in X-rays. This shows that transient brightenings can maintain high temperature plasma only during part of their lifetime. During the emerging and decaying stages of X-ray bright points, the magnetic flux was found to be relatively weak which is a possible reason for not having been detected in the KPNO observations of Golub et al. (1977).

Thanks to the open magnetic flux in coronal holes, plasma at high speed, temperature and density is ejected to far away distances as a result of magnetic reconnection forming X-ray/EUV jets (see Paper III for more discussion). In the present study, the magnetic flux evolution associated with an X-ray jet was presented in unprecedented detail. Similar to X-ray bright points, magnetic cancellation was observed during the eruption. In comparison to a bright point, however, the rate of magnetic cancellation occurring in the footpoints of the X-ray jet was almost twice. Shimojo et al. (1998) analysed the magnetic flux evolution of $25 \mathrm{X}$-ray jets observed by the Soft X-ray telescope onboard Yohkoh using full-disk KPNO longitudinal magnetograms. They found that $72 \%$ of the jets occurred in mixed polarity region. We believe that all brightening events including jets are due to the interaction of bipolar regions. It should be noted that the SOT observations have higher sensitivity and more than ten times higher spatial resolution with respect to the KPNO data. That allowed us to detect much smaller and weaker magnetic flux and as noted earlier, weak magnetic fields are often responsible for the formation of various small-scale transients. More studies based on high-resolution observations of X-ray jet magnetic fields are needed to fully understand their formation patterns.

Following reconnection, the newly formed smaller loops could eventually submerge which is observed as cancellation of magnetic dipoles (Harvey et al. 1999). Many more magnetic cancellation sites than X-ray brightening events were observed in the present paper, i.e. not all magnetic cancellations relate to $\mathrm{X}$-ray brightenings. This result is in agreement with Golub et al. (1977), who found that not all active magnetic regions (namely ephemeral active regions) coincide with X-ray bright points. In order to observe brightening in X-rays, plasma has to be heated to $\mathrm{X}$-ray temperatures, which means that only reconnection sites which release sufficient energy can have an X-ray response.

In Paper III, we have found that transient brightening events have a response over a wide temperature range from the chromosphere to the corona. This result, however, was obtained using spectroscopic EIS and SUMER slit observations, which means that we had a very limited view and cadence to investigate how cancellation of magnetic flux with different strength is related to chromospheric, transition region or/and coronal emission. The multi-wavelength observations from AIA (The Atmospheric Imaging Assembly, Lemen et al. 2012) and HMI (The Helioseismic and Magnetic Imager, Scherrer et al. 2012) on board SDO (The Solar Dynamics Observatory, Pesnell et al. 2012) provide a unique opportunity for further investigation. We should be able to find observational signatures on what is the relation between photospheric magnetic flux cancellation and magnetic reconnection, and whether magnetic cancellation (submergence or other processes) is always related to energy release into the solar atmosphere.

Fisk (2005) modelled the transport of open magnetic fields in the Sun. He suggested that the coronal holes are formed because of a local minimum of dipolar magnetic flux emergence. This idea was later supported from analysing observations from SoHO/MDI (Abramenko et al. 2006) and BBSO (Zhang et al. 2006), where they found dipolar flux emergence rates in coronal holes lower than in quiet-Sun regions. Our study demonstrates that the magnetic flux in coronal holes is continuously processed through magnetic reconnection which is responsible for the formation of numerous small-scale transient events. The open magnetic flux forming the coronal-hole phenomenon is largely involved in these transient features. The question on whether this open flux is transported as a result of the formation and evolution of these transient events, however, still remains open. Additional analysis is needed comprising of field extrapolations from highcadence and high resolution magnetic field observations, together with MHD modelling of magnetic reconnection at coronal holes boundaries and comparison with high-resolution imaging and spectroscopic information. Data from AIA and HMI on SDO may be a suitable first step towards such an analysis. 
Acknowledgements. We gratefully acknowledge the anonymous referee for the very important corrections and constructive suggestions. We thank Miss Kamalam Vanninathan for her careful and critical reading of the manuscript. Research at Armagh Observatory is grant-aided by the N. Ireland Department of Culture, Arts and Leisure (DCAL). The author Z.H. thanks DCAL for the Ph.D. studentship. We also thank STFC for support via grants ST/F001843/1 and PP/E002242/1. The research leading to these results has received funding from the European Commission's Seventh Framework Programme (FP7/20072013) under the grant agreement eHeroes (project no. 284461, http:www. eheroes.eu). The author D.A.L. is supported by NASA grant NNX11AP03G through the Living with a Star program. Hinode is a Japanese mission developed and launched by ISAS/JAXA, with NAOJ as domestic partner, and NASA and STFC (UK) as international partners. It is operated by these agencies in co-operation with ESA and NSC (Norway). SWAMIS was written by Craig DeForest and Derek Lamb at the Southwest Research Institute in Boulder, Colorado. The author MSM thanks ISSI Bern for the support of the team "Magnetic Flux Emergence in the Solar Atmosphere".

\section{References}

Abramenko, V. I., Fisk, L. A., \& Yurchyshyn, V. B. 2006, ApJ, 641, L65 Alexander, C. E., Del Zanna, G., \& Maclean, R. C. 2011, A\&A, 526, A134 Brown, D. S., Parnell, C. E., Deluca, E. E., Golub, L., \& McMullen, R. A. 2001, Sol. Phys., 201, 305

Chae, J., Moon, Y.-J., Park, Y.-D., et al. 2007, PASJ, 59, 619

DeForest, C. E., Hagenaar, H. J., Lamb, D. A., Parnell, C. E., \& Welsch, B. T. 2007, ApJ, 666, 576

Doschek, G. A., Landi, E., Warren, H. P., \& Harra, L. K. 2010, ApJ, 710, 1806 Fisk, L. A. 2005, ApJ, 626, 563

Golub, L., Krieger, A. S., \& Vaiana, G. S. 1976a, Sol. Phys., 49, 79

Golub, L., Krieger, A. S., \& Vaiana, G. S. 1976b, Sol. Phys., 50, 311

Golub, L., Krieger, A. S., Harvey, J. W., \& Vaiana, G. S. 1977, Sol. Phys., 53, 111

Golub, L., Deluca, E., Austin, G., et al. 2007, Sol. Phys., 243, 63

Habbal, S. R. 1992, Ann. Geophys., 10, 34

Habbal, S. R., \& Withbroe, G. L. 1981, Sol. Phys., 69, 77

Habbal, S. R., Withbroe, G. L., \& Dowdy, Jr., J. F. 1990, ApJ, 352, 333

Hagenaar, H. J. 2001, ApJ, 555, 448

Harvey, K. L., Nitta, N., Strong, K. T., \& Tsuneta, S. 1994, in X-ray solar physics from Yohkoh, eds. Y. Uchida, T. Watanabe, K. Shibata, \& H. S. Hudson, 21

Harvey, K. L., Jones, H. P., Schrijver, C. J., \& Penn, M. J. 1999, Sol. Phys., 190, 35

Hundhausen, A. J. 1977, in Coronal Holes and High Speed Wind Streams, ed. J. B. Zirker, 225

Ichimoto, K., Lites, B., Elmore, D., et al. 2008, Sol. Phys., 249, 233

Ito, H., Tsuneta, S., Shiota, D., Tokumaru, M., \& Fujiki, K. 2010, ApJ, 719, 131
Krieger, A. S., Vaiana, G. S., \& van Speybroeck, L. P. 1971, in Solar Magnetic Fields, ed. R. Howard, IAU Symp., 43, 397

Krieger, A. S., Timothy, A. F., \& Roelof, E. C. 1973, Sol. Phys., 29, 505

Lamb, D. A., DeForest, C. E., Hagenaar, H. J., Parnell, C. E., \& Welsch, B. T. 2008, ApJ, 674, 520

Lamb, D. A., DeForest, C. E., Hagenaar, H. J., Parnell, C. E., \& Welsch, B. T. 2010, ApJ, 720, 1405

Lemen, J., Title, A., Akin, D., et al. 2012, Sol. Phys., 275, 17

Madjarska, M. S., \& Wiegelmann, T. 2009, A\&A, 503, 991

Madjarska, M. S., Doyle, J. G., Teriaca, L., \& Banerjee, D. 2003, A\&A, 398, 775

Madjarska, M. S., Doyle, J. G., \& van Driel-Gesztelyi, L. 2004, ApJ, 603, L57

Madjarska, M. S., Huang, Z., Doyle, J. G., \& Subramanian, S. 2012, A\&A, 545, A67

Moore, R. L., Tang, F., Bohlin, J. D., \& Golub, L. 1977, ApJ, 218, 286

Moreno-Insertis, F., Galsgaard, K., \& Ugarte-Urra, I. 2008, ApJ, 673, L211

Parker, E. N. 1988, ApJ, 330, 474

Parnell, C. E., Priest, E. R., \& Titov, V. S. 1994, Sol. Phys., 153, 217

Pérez-Suárez, D., Maclean, R. C., Doyle, J. G., \& Madjarska, M. S. 2008, A\&A, 492, 575

Pesnell, W., Thompson, B., \& Chamberlin, P. 2012, Sol. Phys., 275, 3

Preś, P., \& Phillips, K. H. J. 1999, ApJ, 510, L73

Priest, E. R., Parnell, C. E., \& Martin, S. F. 1994, ApJ, 427, 459

Scherrer, P., Schou, J., Bush, R., et al. 2012, Sol. Phys., 275, 207

Sheeley, Jr., N. R., \& Golub, L. 1979, Sol. Phys., 63, 119

Shibata, K., Ishido, Y., Acton, L. W., et al. 1992, PASJ, 44, L173

Shimojo, M., Shibata, K., \& Harvey, K. L. 1998, Sol. Phys., 178, 379

Subramanian, S., Madjarska, M. S., \& Doyle, J. G. 2010, A\&A, 516, A50

Tian, H., Xia, L.-D., \& Li, S. 2008, A\&A, 489, 741

Tsuneta, S., Ichimoto, K., Katsukawa, Y., et al. 2008, Sol. Phys., 249, 167

Ugarte-Urra, I., Doyle, J. G., Madjarska, M. S., \& O’Shea, E. 2004a, A\&A, 418, 313

Ugarte-Urra, I., Doyle, J. G., Nakariakov, V. M., \& Foley, C. R. 2004b, A\&A, 425, 1083

Vaiana, G. S., Krieger, A. S., Van Speybroeck, L. P., \& Zehnpfennig, T. 1970, Bull. Am. Phys. Soc., 15, 611

van Ballegooijen, A. A., \& Martens, P. C. H. 1989, ApJ, 343, 971

von Rekowski, B., Parnell, C. E., \& Priest, E. R. 2006a, MNRAS, 366, 125

von Rekowski, B., Parnell, C. E., \& Priest, E. R. 2006b, MNRAS, 369, 43

Wang, Y.-M., Sheeley, Jr., N. R., Socker, D. G., et al. 1998, ApJ, 508, 899

Webb, D. F., Martin, S. F., Moses, D., \& Harvey, J. W. 1993, Sol. Phys., 144, 15

Yokoyama, T., \& Shibata, K. 1995, Nature, 375, 42

Zhang, J., Ma, J., \& Wang, H. 2006, ApJ, 649, 464

Zhang, J., Kundu, M. R., \& White, S. M. 2001, Sol. Phys., 198, 347

Zhang, Q. M., Chen, P. F., Guo, Y., Fang, C., \& Ding, M. D. 2012, ApJ, 746, 19

Zwaan, C. 1987, ARA\&A, 25, 83 


\section{Appendix A}

Table A.1. A timeline on the X-ray and magnetic field evolution of a CH BP (marked as No. 1 event in Fig. 1) on 2007 Nov. 9.

\begin{tabular}{|c|c|c|c|}
\hline Time (UT) & $\mathrm{X}$-ray BP behaviour & Magnetic fragment behaviour & Notes \\
\hline $06: 38$ & The BP is already present & A simple bipolar region & Beginning of the dataset \\
\hline $06: 45$ & BP reaches an intensity peak & $\begin{array}{l}\text { A positive fragment moves towards a negative } \\
\text { fragment and magnetic cancellation takes place }\end{array}$ & \\
\hline $06: 57$ & BP becomes weaker & $\begin{array}{l}\text { The cancelling positive fragment } \\
\text { disappears }\end{array}$ & $\begin{array}{l}\text { This scenario repeats } \\
\text { around every } 25 \text { minutes on average. The } \\
\text { fluctuation always relates to magnetic } \\
\text { cancellation and the cancellation site is } \\
\text { always near to major positive polarities, } \\
\text { but sometimes only negative fragments } \\
\text { move to the site and at other times both } \\
\text { polarities move and join together. }\end{array}$ \\
\hline $07: 52$ & & $\begin{array}{l}\text { Negative fragments start to } \\
\text { emerge at about } 10^{\prime \prime} \text { away } \\
\text { from the positive ones, then } \\
\text { start moving towards the positive } \\
\text { polarities. }\end{array}$ & \\
\hline $08: 22$ & $\begin{array}{l}\text { A small BP emerges at the } \\
\text { west side. }\end{array}$ & $\begin{array}{l}\text { A few weak negative fragments emerge west } \\
\text { of the domain positive fragments } \\
\text { and cancellation occurs between these } \\
\text { two polarities. }\end{array}$ & \\
\hline $08: 43$ & The small BP disappears. & $\begin{array}{l}\text { One positive fragment at the west part } \\
\text { disappeared after cancellation with newly } \\
\text { emerging negative fragments. }\end{array}$ & \\
\hline 09:45 & $\begin{array}{l}\text { The main BP reaches its second } \\
\text { highest intensity peak. }\end{array}$ & $\begin{array}{l}\text { The emerged negative fragments at } 07: 52 \text { move } \\
\text { closer to positive polarities and cancellation occurs. }\end{array}$ & $\begin{array}{l}\text { A few negative fragments disappear after } \\
\text { this time and that leads to } \\
\text { the BP fading again }\end{array}$ \\
\hline $10: 10$ & $\begin{array}{l}\text { The main BP reaches its } \\
\text { highest intensity peak. }\end{array}$ & $\begin{array}{l}\text { The rest of the emerged negative fragments keep } \\
\text { moving towards the positive polarities and cancel. }\end{array}$ & $\begin{array}{l}\text { Again it fades after the disappearance of a } \\
\text { few negative fragments, however existing } \\
\text { negative fragments keep cancelling and } \\
\text { totally disappear at 11:25 UT creating } \\
\text { a few peaks in the lightcurve of the BP. }\end{array}$ \\
\hline $10: 36$ & $\begin{array}{l}\text { A new BP emerges southwest } \\
\text { of the main one. }\end{array}$ & $\begin{array}{l}\text { Another negative polarity to the southwest } \\
\text { move towards the positive polarities. }\end{array}$ & $\begin{array}{l}\text { Cancellation and disappearance of this } \\
\text { group of negative polarities take control } \\
\text { of the behaviour of the new born BP. }\end{array}$ \\
\hline $12: 31$ & BP is almost fully gone. & $\begin{array}{l}\text { Both positive polarities and the southwest } \\
\text { negative polarities move towards each other, } \\
\text { however, no cancellation was visible. }\end{array}$ & $\begin{array}{l}\text { The main BP is very weak but still visible } \\
\text { When some other negative fragments } \\
\text { move closer to the positive fragments, } \\
\text { the BP flames up again until the end of the } \\
\text { dataset, the positive polarities still } \\
\text { exist, and the BP repeats its intensity } \\
\text { fluctuation. }\end{array}$ \\
\hline
\end{tabular}


Z. Huang et al.: Coronal hole boundaries at small scales. IV.
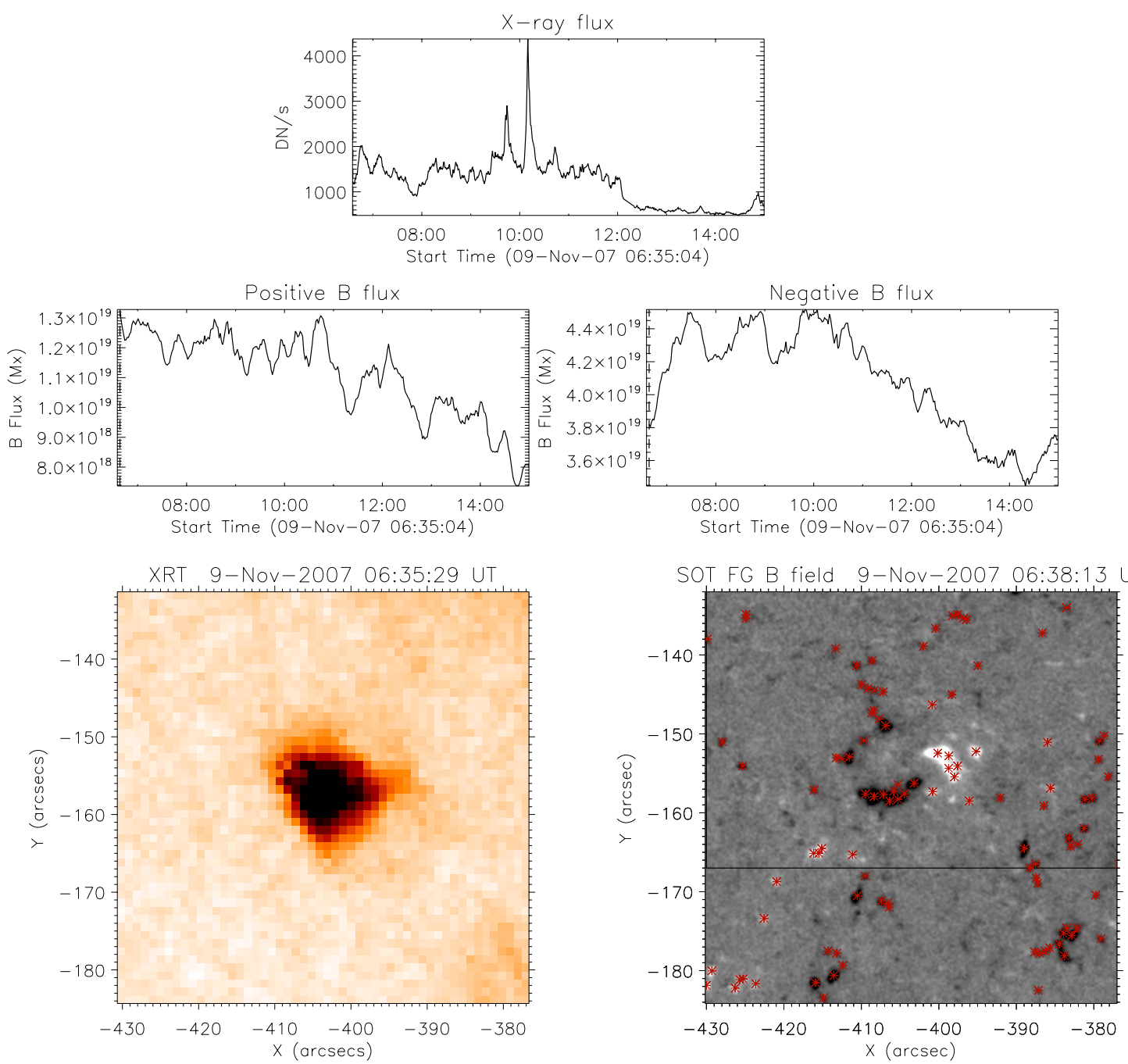

Fig. A.1. Animation showing evolution of CH event No. 1 on 2007 November 9 (Figs. 6 and 7). The top panel is the X-ray lightcurve; the middle panel is the lightcurves of positive (left) and negative (right) longitudinal magnetic flux; the bottom panel shows the evolution of the event seen in X-ray images (left) and SOT FG longitudinal magnetograms (right). The time axis in the lightcurve plots is showen in UT. During the animation, the dashed line in the lightcurve indicates the given time of the X-ray image and longitudinal magnetogram (bottom panel). Solid lines in the magnetograms outline the region where the magnetic flux lightcurves were extracted. Symbols indicate magnetic features tracked by SWAMIS. Asterisks are features first detected at the given time. Cross symbols indicate magnetic features tracked from the past. Square symbols indicate magnetic features first detected and defined by SWAMIS as flux emergence. (Please note that in this figure only asterisks are shown because it is the first frame of the observation, and other symbols will present through the animation.) 
A\&A 548, A62 (2012)
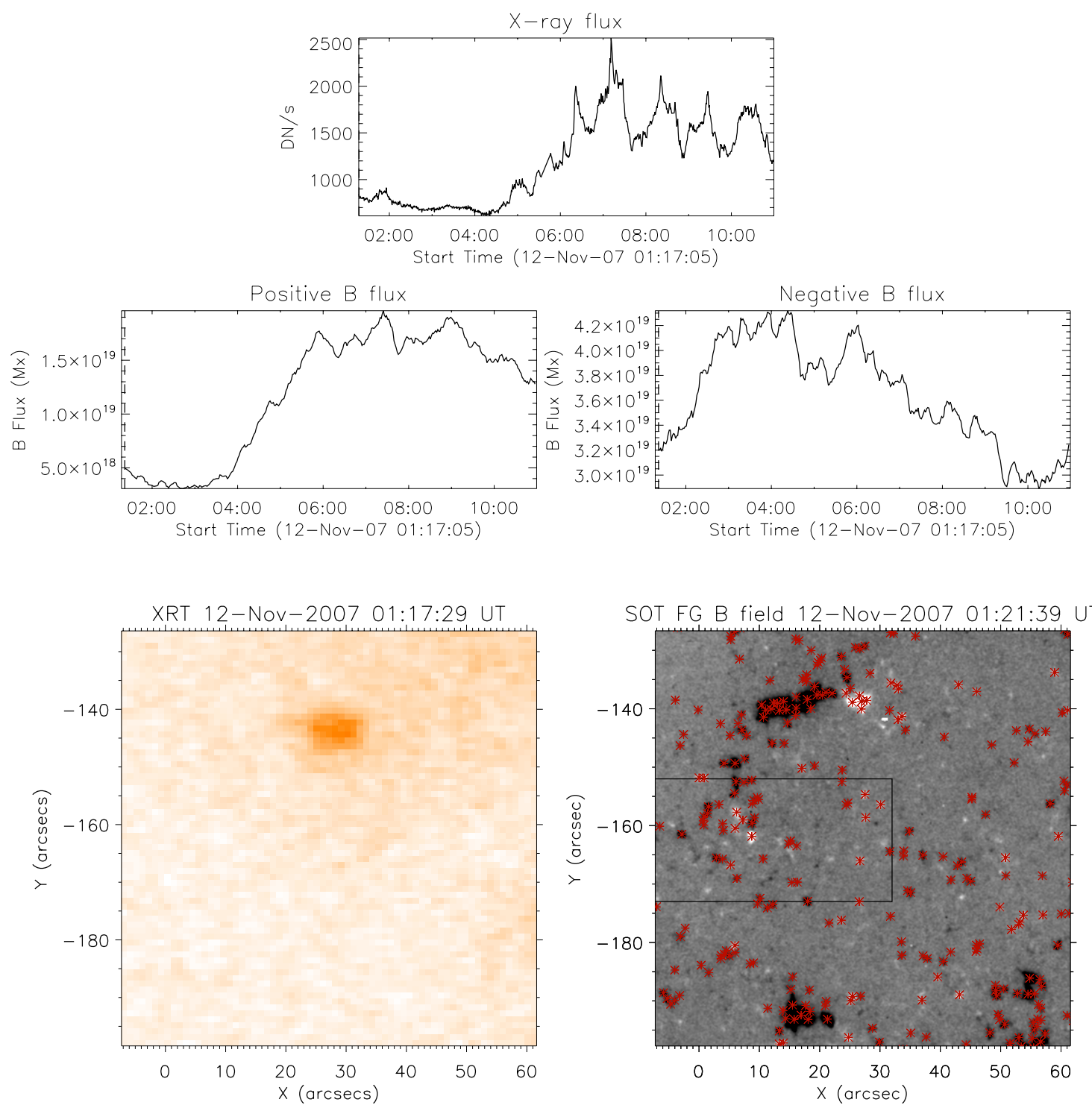

Fig. A.2. As Fig. A.1, but for CH brightening event No. 2 on 2007 November 12 (Figs. 9 and 10). 
Z. Huang et al.: Coronal hole boundaries at small scales. IV.
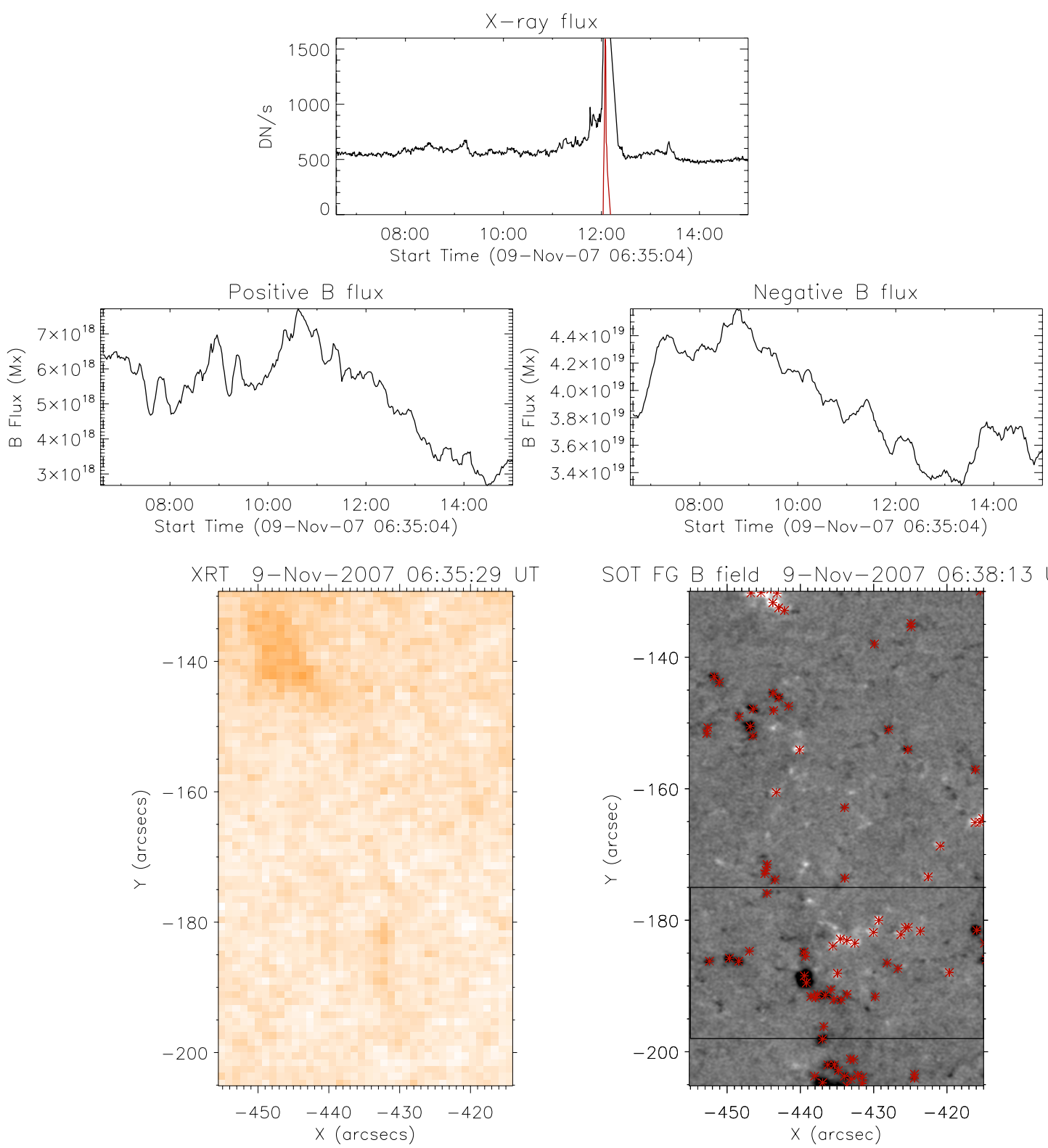

SOT FG B field 9-Nov-2007 06:38:13 U

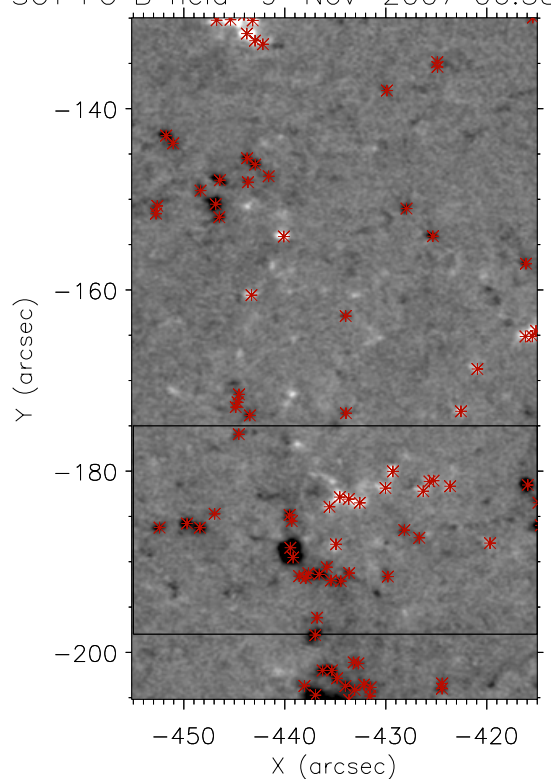

Fig. A.3. As Fig. A.1, but for the CH X-ray jet, No. 3 on 2007 November 9 (Figs. 13 and 14). 